\title{
Possible Role of Dendritic Compartmentalization in the Spatial Working Memory Circuit
}

\author{
Kenji Morita \\ RIKEN Brain Science Institute, Wako 351-0198, Japan
}

In spatial working memory tasks, pyramidal cells in the relevant cortical circuit receive massive inputs to shape stimulus locationselective sustained activity. A significant part of those inputs are applied onto the dendrites. Considering the dendritic morphology and circuit anatomy together with recently suggested branch-specific plasticity rules, external inputs transmitting the information of the stimulus location would be mapped onto some portion of the dendritic branches, rather than uniformly distributed over the branches. Meanwhile, recent studies revealed that each dendritic branch of pyramidal cell functions as a compartmentalized integration subunit through local spike generation and branch-specific excitation-inhibition interaction. I have examined how such nonlinear dendritic integration, combined with the nonuniform distribution of the external input, affects the behavior of the whole circuit by constructing a rate-coding model incorporating multiple dendritic branches of the individual pyramidal cell. Simulations varying the nature of dendritic nonlinearity and the configuration of somatically and dendritically mediated recurrent inhibition revealed that dendritic compartmentalization potentially enables the circuit to form an accurate memory depending on the contrast of the external input, but insensitively to its intensity, under certain conditions; in particular, when there exists tuned global dendritic recurrent inhibition or local dendritic inhibition coupled with global somatic inhibition. The model suggests that, when the circuit receives low-contrast or background input, only a small portion of dendritic branches of each pyramidal cell can overcome the local threshold so as to contribute to the somatic low-frequency firing, which in turn stabilizes the low-activity state of the circuit by recruiting recurrent inhibition.

Key words: dendritic arborization; local circuit; recurrent inhibition; sustained activity; working memory; decision making; dendritic inhibition; microcircuit; neural network model; dendrite; spatial

\section{Introduction}

In working memory tasks, pyramidal cells in the relevant cortical circuit receive feedforward sensory inputs and recurrent inputs to shape stimulus-selective sustained activity. To reveal cellular mechanisms of working memory, it seems essential to understand how different types of inputs are integrated in individual pyramidal cells. Most excitatory inputs are applied onto the pyramidal dendrites. Regarding inhibition, although some types of inhibitory interneurons almost exclusively target cell bodies or axon initial segments of the pyramidal cells, many types target the dendrites, including the distal parts and the spines (Kawaguchi and Kubota, 1997; Somogyi et al., 1998; Markram et al., 2004). Although detailed distribution of inhibitory, as well as excitatory, inputs on the dendrites of the pyramidal cells in the working memory circuit remains elusive, existence of dendritically mediated recurrent inhibition has been suggested (González-Burgos et al., 2005).

Pyramidal dendrites have multiple branches, and synaptic in-

\footnotetext{
Received Jan. 7, 2008; revised May 29, 2008; accepted June 17, 2008.

This work was supported by a Grant-in-Aid for Young Scientists (B) 19700310 from the Ministry of Education, Culture, Sports, Science, and Technology of Japan. I thank Dr. S. Amari for helpful discussion. I also thank anonymous reviewers for their valuable comments.

Correspondence should be addressed to Dr. Kenji Morita, RIKEN Brain Science Institute, 2-1, Hirosawa, Wako 351-0198, Japan. E-mail: morita@brain.riken.jp.

DOI:10.1523/JNEUROSCI.0059-08.2008

Copyright $\odot 2008$ Society for Neuroscience $\quad$ 0270-6474/08/287699-26\$15.00/0
}

tegration thereon has been extensively studied (Rall, 1964; Koch et al., 1983; Segev and Parnas, 1983; Shepherd and Brayton, 1987; Mel, 1993; Segev, 1995; London and Häusser, 2005). An emerging view, originally advanced on theoretical grounds (Koch et al., 1983; Shepherd and Brayton, 1987; Poirazi et al., 2003a,b; Mel, 2007) but now supported by a considerable amount of experimental results (Häusser and Mel, 2003; Spruston et al., 2007), is that individual dendritic branches can operate as computational subunits, each of which implements compartmentalized nonlinear input integration by virtue of dendritic spike generation (Schiller et al., 2000; Polsky et al., 2004; Losonczy and Magee, 2006; Nevian et al., 2007) and branch-specific inhibition (Liu, 2004; Rhodes, 2006). Such dendritic compartmentalization in individual neurons can generally affect the collective behavior of the network activity (Spratling and Johnson, 2001, 2002; Goldman et al., 2003; Morita et al., 2007), and thus appears likely to affect the computational property of the cortical circuit for working memory.

Among various types of working memory, spatial working memory is one of the best studied systems. Based on the experimental results (Funahashi et al., 1989), fundamental organization of the underlying cortical circuit has been proposed (Goldman-Rakic, 1995) wherein the pyramidal cells with similar selectivity strongly excite each other to sustain their activity and simultaneously inhibit other cells via inhibitory interneurons. By incorporating such a basic network architecture, numerous mod- 
eling studies have elaborated the detailed biological mechanisms (Camperi and Wang, 1998; Compte et al., 2000; Renart et al., 2003; Constantinidis and Wang, 2004; Carter and Wang, 2007). However, thus far, possible effects of pyramidal dendritic compartmentalization have not been examined. Although some studies considered the pyramidal dendrites (Tegnér et al., 2002; Wang et al., 2004), they did not consider multiple branches. In this study, I have addressed this issue by constructing a neural circuit model incorporating pyramidal dendritic arborization and branch-specific nonlinear input integration. Through simulations, I show that dendritic compartmentalization potentially endows the circuit with the ability to form an accurate spatial memory depending on the contrast of the external feedforward input to the circuit, but insensitively to its intensity. I describe desired conditions, explore the underlying mechanism so as to derive testable predictions, and discuss possible behavioral relevance.

\section{Materials and Methods}

Network architecture. I assumed that a neuronal circuit for spatial working memory exists in the cerebral cortex (Goldman-Rakic, 1995). The concerned excitatory pyramidal cells (see Fig. $1 A-E, G$, pyramids at bottom) were presumed to receive feedforward excitation (see Fig. $1 A$, red arrows) from the neurons in the preceding brain areas such as higherorder visual cortices. These "input layer neurons" (see Fig. $1 A-E, G$, circles at top) were assumed to have their own "preferred angles" (see Fig. $1 A-E, G$, arrows in the circles); thus, each neuron becomes transiently active on stimulus presentation at an angle that is close to its preferred angle. I referred to the population activity of these input layer neurons as the "input to the memory circuit" or the "external/feedforward input" (see Fig. $1 A$, orange bars at top), which therefore peaks around the stimulus angle. Through the feedforward connectivity that was assumed to be topographically organized in a functional sense, but not necessarily in a physical sense, the pyramidal cells in the memory circuit also have their own preferred angles (see Fig. $1 A-E, G$, arrows in the pyramids) (for more details, see below). Between the pyramidal cells, recurrent excitation via axonal collaterals (see Fig. $1 B$ ) and recurrent inhibition either via soma-targeting interneurons (see Fig. 1C) or via dendrite-targeting interneurons (see Fig. 1D) were assumed to exist (single cell population would target both the soma and the dendrite) (see below). Recurrent excitation was assumed to be organized such that the strength of synaptic coupling between two pyramidal cells decreases with the difference in their preferred angles (for details, see below). Somatically mediated recurrent inhibition was assumed to be unstructured (global). Dendritically mediated recurrent inhibition was assumed to be either global or local (see below). Inhibitory interneurons were not explicitly modeled but instead represented by negative signs preceding the connection strengths. Although soma-targeting and dendrite-targeting interneurons are schematically illustrated as separate cell populations in the figures, it might be more likely that a single cell population could implement both of them. Somatically mediated global recurrent inhibition could be implemented by large basket cells as assumed in a previous study (Wang et al., 2004). As for dendritically mediated recurrent inhibition, there seem to be many possibilities, given that there is a huge variety of neocortical GABAergic interneurons (Kawaguchi and Kubota, 1997; Somogyi et al., 1998; Markram et al., 2004) and most of them target pyramidal dendrites with various degree of preference [Kubota et al. (2007), their Fig. 4]. Among them, I would propose that basket cells (Gupta et al., 2000; Wang et al., 2002), at least certain subtypes of them, can also implement the dendritic (in addition to somatic) recurrent inhibition such as assumed in the model of the present study, despite their being known as somatargeting cells. Basket cells target pyramidal somata, because it is a defining feature, but they also generally target proximal dendrites, which would include the whole basal dendrite; it was shown that at least certain subtypes of basket cells prevalently target medial or even distal portion of pyramidal basal dendrites [Wang et al. (2002), their Fig. 5, for the nest basket cell; the most preferable target cite was the third-order basal dendritic branches; or Gupta et al. (2000), their Fig. 6C, for the small basket cell] (however, it was also shown that at least some basket cells made synapses exclusively on the soma or the most proximal basal dendrites [Tamás et al. (1997), their Fig. 2C]); whether or not to target medial/ distal basal branches therefore seems to depend on the subtype, brain region, etc. (Kubota et al., 2007). Recent studies have revealed that local dendritic spikes (NMDA spikes) can be generated independently in each branch of the pyramidal basal dendrites at a location $>70 \mu \mathrm{m}$ away from the soma (Schiller et al., 2000; Polsky et al., 2004; Nevian et al., 2007), and it was thus suggested (Nevian et al., 2007) that basal dendrites, despite their proximity to the soma, do not form a single basal-somatic region but rather should be considered as a separate integrative compartment. Although a direct experiment regarding the effect of inhibitory input applied onto the pyramidal basal dendrite with single branch resolution has not so far been done to my knowledge, a recent detailed simulation study (Rhodes, 2006) has shown that inhibitory input applied onto the basal dendritic branch close to $(\sim 20 \mu \mathrm{m})$ the cite of NMDA spike generation (or more distal toward the end of the branch) vetoes the NMDA spikes in a branch-specific manner. The average distance of the basketto-pyramidal synapses from the pyramidal cell body was shown to be $97.06 \pm 41.49,68.28 \pm 13.14$, or $78.83 \pm 20.61 \mu \mathrm{m}$ for the large, nest, and small basket cells, respectively [Markram et al. (2004), their supplemental information S2], and therefore it seems likely that certain portion of the synapses made by those cells onto the pyramidal basal dendrites (and possibly also proximal apical/oblique dendrites) can perform the branch-specific veto operation as mentioned above. In the case in which excitatory inputs are rather weak and temporally and/or spatially dispersed, NMDA spikes would not be generated and location-independent summation of the excitatory inputs would instead occur (Nevian et al., 2007) (see also Cash and Yuste, 1999; Gasparini and Magee, 2006); however, inhibition applied onto the branch would still have a branchspecific effect in such a subthreshold regimen via shunting, as theoretically predicted (Koch et al., 1983; Shepherd and Brayton, 1987) and experimentally demonstrated (Liu, 2004) (although not specifically in the neocortical pyramidal basal dendrites but in the cultured hippocampal neurons). Regarding the distal apical dendrites, although basket cells would also make synapses on the apical, oblique, and even tuft dendrites (e.g., it was shown that axodendritic synapses made by nest basket cells are formed onto three-quarters of the pyramidal dendritic tree [Wang et al. (2002), their Fig. 5]), other types of interneurons, such as (subtypes of) bitufted cells (Markram et al., 2004) or calbindin-positive interneurons with ascending axons observed in the monkey prefrontal cortex (Zaitsev et al., 2005), for example, would implement branch-specific inhibition on the distal apical/tuft dendrites. Martinotti cells, the most well-known cell type known to target pyramidal apical/tuft dendrites (Kawaguchi and Kubota, 1997; Markram et al., 2004), are however not considered to be a candidate for the source of such dendritic inhibition as assumed in this study, because it was shown that pyramidal-to-Martinotti synapses show prominent short-term facilitation, different from many other pyramidal-to-interneuron synapses, so that the Martinotti cell becomes active only after it receives high-frequency input from a single presynaptic pyramidal cell (Kapfer et al., 2007; Silberberg and Markram, 2007), which seems incompatible with the model of the present study (for details, see Results, On the nature of recurrent inhibition). By virtue of those excitatory and inhibitory recurrent connections, it is expected that a small portion of the pyramidal cells whose preferred angles are close to the stimulus angle sustain their activity after stimulus extinction while the others are inactivated, thereby representing the memory of the stimulus angle (Goldman-Rakic, 1995).

Distributions of the inputs on the dendritic branches. I considered a model of individual pyramidal cells with multiple dendritic branches (see Fig. 1). As for the distribution of the synaptic inputs over the branches, I have made the following assumptions, based on recent experimental findings about the morphology, plasticity, and circuit anatomy regarding the pyramidal cellular dendrites (for explanation, see Results). I assumed that different dendritic branches of a given pyramidal cell receive feedforward (external) inputs from different input layer neurons (see Fig. $1 G$ ). For the sake of simplicity, I further assumed the one-to-one connectivity as shown in the figure; however, I have confirmed that such an exact one-to-one connectivity seems not to be important for the main 
results of the present study (see Fig. 9). The connection strength between each dendritic branch of a given pyramidal cell and the input layer neuron connected to the branch was assumed to have a bell-shaped distribution peaked at the connection to the input layer neuron with the same angle preference as the pyramidal cell (see Fig. 1G, orange line). Note that, because of this assumption, effectively only some portion of the dendritic branches receives a significant amount of feedforward inputs whereas other branches receive little feedforward excitation. In other words, almost all the feedforward inputs converge onto some portion of dendritic branches. Recurrent excitation, and recurrent inhibition mediated by dendrite-targeting interneurons when it was considered, was assumed to be evenly applied onto all the dendritic branches in most simulations, although nonuniformly distributed recurrent excitation (see Fig. $1 G$, red dashed line) was also tested in some simulations (see Fig. $13 D-F$ and the corresponding text in Results). If intradendritic input distributions are quite different from those assumed ones, results (circuit behaviors) may differ. Nevertheless, I would like to argue that the main point of the present study, namely, a feature of the circuit behavior (see Results), seems not to be a direct outcome of the particular intradendritic input distributions as assumed above, but rather a more generic phenomenon, because similar feature appeared in a related simpler model without such an assumption (Morita et al., 2007) (although that model included only self-excitation, but not mutual excitation). More specifically, now let us consider the simplest situation, for the sake of explanation, in which the dendritic threshold is 0 , dendritic saturation is absent, and dendritic inhibition operates as a subtraction. If all the inputs, namely, feedforward excitation, recurrent excitation, and recurrent inhibition have exactly the same intradendritic distribution, it is equivalent to have only a single branch because relative ratios of each type of inputs between the branches are constant all the time (i.e., activity of every branch changes proportionally; for example, thresholding is simultaneously imposed in every branch). However, if the distributions are different between input types, thresholding is expected to be imposed at different times from branch to branch, and thus, an effect of having multiple branches could appear. How strongly such an effect appears, as well as what type of effect appears, would depend on the particular shapes of the distributions. In the present study, I have assumed bell-shaped external excitation, uniform or weakly bell-shaped recurrent excitation, and uniform recurrent inhibition, based on the biological findings as mentioned above, and have found that a particular feature appears under such an assumption (see Results). A similar feature, as well as another feature yet to be known, might appear, at least theoretically, under different intradendritic distributions.

Dynamics of the pyramidal cellular activity. Let us denote the activity (firing rate) of a pyramidal cell with preferred angle (memory/response field) $\theta$ at time $t$ by $x(t, \theta)$. I described the temporal evolution of activities of the pyramidal cells by the following set of differential equations:

$$
\begin{aligned}
& \frac{d x(t, \theta)}{d t}=-x(t, \theta)+\sigma_{s}\left(\sum_{\varphi} \sigma_{d}(J(\varphi, \theta))-a_{s} S(t)\right), \theta \in \\
& \quad\left[-180^{\circ} 180^{\circ}\right), \varphi \in\left[-180^{\circ} 180^{\circ}\right) .
\end{aligned}
$$

Here, $J(\varphi, \theta)$ represents the total input activity, which comprises feedforward excitation, recurrent excitation, and dendritically mediated recurrent inhibition (see below), on the dendritic branch of the pyramidal cell with preferred angle $\theta$, which receives feedforward excitation from the input layer neuron with the preferred angle $\varphi . S(t) \equiv \Sigma_{\theta^{\prime}} x\left(t, \theta^{\prime}\right)$ is the summation of the activities of all the pyramidal cells, and the negative term $-a_{s} S(t)$ represents somatically mediated recurrent inhibition with $a_{s}$ representing the strength. The function

$$
\sigma_{d}(z)=\left\{\begin{array}{cc}
0 & \left(z<\beta_{d}\right) \\
\alpha_{d}\left(z-\beta_{d}\right) & \left(\beta_{d} \leq z<\eta_{d}\right) \\
\eta_{d} & \left(\beta_{d}+\frac{\eta_{d}}{\alpha_{d}} \leq z\right)
\end{array}\right.
$$

represents the nonlinear input integration on each dendritic branch (see Fig. $1 \mathrm{Ha}) ; \beta_{d}$ is the dendritic threshold; $\alpha_{d}$ is the slope; and $\eta_{d}$ is the upper bound (saturation). The function

$$
\sigma_{s}(z)=\left\{\begin{array}{cc}
0 & (z<0) \\
z & (0 \leq z)
\end{array}\right.
$$

represents the threshold nonlinearity imposed on the summation of all the branch outputs and somatic inhibition at the soma. This represents the transformation from the input activity to the output firing rate through spike generating mechanisms at the soma. The threshold nonlinearity would correspond to that the neuron starts firing just when the mean current averaged over a short time duration it receives is equal to zero, although our model is based on the firing rate description so that such a direct interpretation might not be appropriate. It has been shown that, in the case of pyramidal cells receiving a mixture of fluctuating excitatory and inhibitory inputs, the minimum mean current necessary for firing would be around zero, or could be slightly negative, because spikes can be driven by the fluctuation of the current (Hô and Destexhe, 2000; Chance et al., 2002; Shu et al., 2003b). The main findings in the present study continue to hold at least to a certain extent with a negative somatic threshold (data not shown). However, if the somatic threshold is positive, competition between pyramidal cells is further promoted so that inaccurate memory would become more easily induced even with compartmentalized input integration in the dendritic branches (data not shown).

Inputs onto each dendritic branch. As described above, the pyramidal cells (see Fig. $1 A-E, G$, bottom pyramids) were assumed to be conceptually, but not necessarily physically, distributed in a ring according to their own preferred angles $\left(\theta ;-180^{\circ} \leq \theta<180^{\circ}\right.$; indicated by arrows in the pyramids in Fig. $1 A-E, G)$. They were assumed to receive feedforward excitation from the input layer neurons in the preceding brain area (see Fig. $1 A-E, G$, top circles). The activity of the input layer neuron with preferred angle $\varphi$ (see Fig. $1 A-E, G$, arrow in the circles) under the presentation of the visual stimulus at the angle $\varphi_{0}$, denoted by $I\left(\varphi ; \varphi_{0}\right)$, was assumed to be the sum of the following three components:

$$
I\left(\varphi ; \varphi_{0}\right)=I_{0}+I_{s}\left(\varphi ; \varphi_{0}\right)+\xi(\varphi)
$$

where (1) $I_{0}$ is the background activity, (2) $I_{s}\left(\varphi ; \varphi_{0}\right)$ is the "stimulus angle-correlated component" that depends on the location, or angle, of the presented visual stimulus $\left(\varphi_{0}\right)$ and was assumed to be represented by a bell-shaped function centered at $\varphi_{0}$ with the height $I_{s \text { max }}$, specifically, the following:

$$
I_{s}\left(\varphi ; \varphi_{0}\right)=I_{\text {smax }} \exp \left(\left(\cos \left(\varphi-\varphi_{0}\right)-1\right) / \delta_{\text {input }}^{2}\right),
$$

and $(3) \xi(\varphi)$ is Gaussian random noise satisfying $\sqrt{\operatorname{Var}(\xi)}=\varepsilon l_{0}(\varepsilon$, noise level). In most simulations, $\xi(\varphi)$ was not assumed to change with time (static noise). However, I have also performed simulations with time-varying noise [dynamic noise, $\xi(t, \varphi)$ ] by applying independent Gaussian noise at every time step of the simulation (fourth-order RungeKutta method), confirming that main results mostly did not change (see Fig. 14). Note that, because the model describes the dynamics of the firing rate in an abstract manner, these noises do not directly correspond to biophysical processes. The ratio of the stimulus angle-correlated component to the background $\left(I_{s \max } / I_{0}\right)$ is referred to as an "input contrast" (see Fig. $2 \mathrm{Ba}$ ). Feedforward excitatory connections (see Fig. $1 \mathrm{~A}$, red arrows) were assumed to be topographically organized in a functional sense, but not necessarily in a physical sense, such that the input layer neuron and the pyramidal cell with similar preferred stimulus angles have strong connections. Specifically, it was represented as follows:

$$
F(\varphi, \theta)=F_{\max } \exp \left(\cos (\varphi-\theta)-1 / \delta_{f}^{2}\right),
$$

where $F_{\max }$ and $\delta_{f}$ represent the maximum strength and the width of the convergence-divergence tuning of the connection, respectively. Recurrent excitation (see Fig. $1 B$, red arrows) was organized such that the strength of synaptic coupling between two pyramidal cells decreases with the difference in their preferred angles. Specifically, the strength of the recurrent excitation between two pyramidal cells with preferred angles $\theta$ and $\theta^{\prime}$ was described as follows:

$$
E\left(\theta, \theta^{\prime}\right)=E_{\max } \exp \left(\cos \left(\theta-\theta^{\prime}\right)-1 / \delta_{\mathrm{re}}^{2}\right),
$$


where $E_{\max }$ and $\delta_{\text {re }}$ represent the maximum strength and the width of the connection tuning, respectively. Although recurrent excitation was assumed to be uniformly distributed over branches in most simulations, nonuniformly distributed recurrent excitation was also tested in some simulations (see Fig. 13D-F and the corresponding text in Results). In those cases, recurrent excitation from the neuron $\theta^{\prime}$ to the neuron $\theta$ was assigned more onto the branch that receives feedforward input from the input layer neuron with the same angle preference $\theta^{\prime}$ (see Fig. $1 G$, red dashed line) according to the following:

$$
E\left(\theta, \theta^{\prime}\right)\left(c_{1}+c_{2} \exp \left(\cos \left(\psi-\theta^{\prime}\right)-1 / \delta_{\text {re_branch }}^{2}\right)\right) / c_{3},
$$

where $\psi$ represents the preferred angle of the input layer neuron connected to each branch and $\delta_{\text {re } b \text { branch }}$ represents the range (width) of such nonuniformity of recurrent excitation. In the simulations, I used $c_{1}=1$, $c_{2}=0.5, \delta_{\text {re_branch }}=\pi / 4$ (see Fig. $1 G$, red dashed line). $c_{3}$ is a normalization factor:

$$
c_{3}=1 /\left(c_{1}+c_{2} \exp \left(\cos \left(\psi-\theta^{\prime}\right)-1 / \delta_{\text {re_branch }}^{2}\right)\right) .
$$

Dendritically mediated recurrent inhibition (see Fig. $1 D$, blue arrows) was assumed to be either global:

$$
H(t)=a_{d} S(t)
$$

where $a_{d}$ represents the strength, or local in a similar manner to the recurrent excitation (see Fig. $11 \mathrm{~A}$ ):

$H(t)=\sum_{\theta^{\prime}} D\left(\theta, \theta^{\prime}\right) x\left(t, \theta^{\prime}\right)$ with $D\left(\theta, \theta^{\prime}\right)=D_{\max } \exp \left(\cos \left(\theta-\theta^{\prime}\right)\right.$

where $D_{\max }$ and $\delta_{\mathrm{ri}}$ represent the maximum strength and the width of the connection tuning, respectively. In some simulations (see Fig. 13B,C), positive threshold for dendritic recurrent inhibition was considered as follows:

$$
H(t)=\left[\sum_{\theta^{\prime}} D\left(\theta, \theta^{\prime}\right) x\left(t, \theta^{\prime}\right)-c_{\mathrm{ri}}\right]^{+},
$$

where $c_{\mathrm{ri}}$ represents the threshold (set to 0.05) and the superscript "plus" means rectification. As for the effect of dendritically mediated inhibition, I considered two cases: subtraction from the excitatory inputs or division on the excitatory inputs (see Fig. $1 \mathrm{Hb}, \mathrm{Hc}$ and the corresponding text in Results). Note that inhibitory interneurons were not explicitly modeled but instead represented by negative signs in both cases. The total input activity on a dendritic branch was thereby the sum of feedforward excitation and recurrent excitation, subtracted or divided by dendritically mediated recurrent inhibition:

$$
J(\varphi, \theta)=F(\varphi, \theta) I\left(\varphi ; \varphi_{0}\right)+\sum_{\theta^{\prime}} E\left(\theta, \theta^{\prime}\right) x\left(t, \theta^{\prime}\right)-H(t),
$$

or

$$
J(\varphi, \theta)=\frac{F(\varphi, \theta) I\left(\varphi ; \varphi_{0}\right)+\sum_{\theta^{\prime}} E\left(\theta, \theta^{\prime}\right) x\left(t, \theta^{\prime}\right)}{1+H(t)} .
$$

Simulation. In the simulations, I assumed $n$ pyramidal cells and $m$ dendritic branches per pyramidal cell, whose preferred angles are evenly spaced in $-180^{\circ} \leq \theta<180^{\circ}$ and $-180^{\circ} \leq \varphi<180^{\circ}$. In most simulations, I set $n=100$ and $m=100$, except for those varying the number of branches (see Results) (see Fig. 9). Visual stimulus was assumed to appear at time 0 and disappear at time 100 (for the simulations in Figs. 3, 4, $9 \mathrm{Bb}$, and $10 \mathrm{~B}$ : the cases with "moderately nonlinear dendrite") (see below) or at time 200 (for others: the cases with "highly nonlinear dendrite"). Probability of memory formation and the accuracy was calculated at time 200 (for the simulations in Figs. 3, 4, 9Bb and $10 \mathrm{~B}$ ) or at time 400 (for others). Other parameters were set as follows, unless otherwise mentioned. Common to all the cases were the following: $\delta_{\text {input }}=$ $15^{\circ}, F_{\max }=1, \delta_{f}=15^{\circ}, E_{\max }=15, \delta_{\mathrm{re}}=15^{\circ}, \eta_{d}=\frac{1}{\mathrm{~m}}$, and $\varepsilon=0.1$.

Different values for the strength of recurrent excitation $E_{\max }$ were also examined in some simulations: see Figure 13, $E$ and $F$ (see below for more details about the parameter dependence). I considered two different types of dendritic nonlinearity, which I refer to as the moderately nonlinear dendrite (see Fig. $1 \mathrm{Hb}$ ) and the highly nonlinear dendrite (see Fig. $1 \mathrm{Hc}$ ), respectively (see Results). Parameters for the moderately nonlinear dendrite are as follows: $\alpha_{d}=1, \beta_{d}=0, I_{0}=0.1, a_{s}=2$ in the case of somatic inhibition (see Figs. 3, 5) or $a_{d}=\frac{2}{m}$ in the case of dendritic inhibition (see Figs. 4, 5), unless otherwise described. Parameters for the highly nonlinear dendrite are as follows: $\alpha_{d}=5, \beta_{d}=\frac{0.1}{m}$, and $a_{s}=5$ in the case of somatic inhibition or $a_{d}=12$ in the case of global dendritic inhibition (see Figs. 6, 7), unless otherwise described. In the simulations in which the threshold of the moderately nonlinear dendrite was subject to random positive deviation (see Fig. $10 \mathrm{~B}$ ), the threshold $\beta_{d}$ was assumed to be distributed according to the following half-Gaussian distribution (see Fig. 10Aa):

$$
P(x)=\frac{1}{2 \sqrt{2 \pi} \sigma} \exp \left(-\frac{x^{2}}{2 \sigma^{2}}\right)(x \geq 0),
$$

where $\sigma$ (SD of the original Gaussian) was set to 0.02 (see Fig. $10 \mathrm{Aa}$, green curve) (results are shown in Fig. $10 \mathrm{Bb}$ ) or 0.04 (see Fig. $10 \mathrm{Aa}$, blue curve) (results are shown in Fig. $10 \mathrm{Bc}$ ). In the simulations in which the threshold $\left(\beta_{d}\right)$, slope $\left(\alpha_{d}\right)$, and upper bound $\left(\eta_{d}\right)$ of the highly nonlinear dendrite were subject to random variations (see Fig. 10C), they were assumed to be distributed according to the Gaussian distributions whose SDs were set to 0.1 or 0.2 times the means $\left(\frac{0.1}{m}, 5\right.$, and $\frac{1}{m}$ for $\beta_{d}, \alpha_{d}$, and $\eta_{d}$, respectively) (see Fig. $10 A b, A c$ ) (results are shown in Fig. $10 C a, C b$ ) or varied from 0 to 0.2 of the means (see Fig. $10 C e, C f$ ). In the simulations in which locally operated dendritic recurrent inhibition was considered (see Figs. 11-13), the peak strength $\left(D_{\max }\right)$ was varied as described in the figures. The width $\left(\delta_{\text {ri }}\right)$ was set to $45^{\circ}$ (see Fig. $\left.11 A a\right), 30^{\circ}(A b), 22.5^{\circ}$ $(A c)$, or $15^{\circ}(A d)$ (see Results). In the simulations in which both global somatic recurrent inhibition and global or local dendritic inhibition were considered (see Figs. 12,13), the values for $a_{s}, a_{d}, D_{\max }$, and $\delta_{\text {ri }}$ were varied as described in the figures. The initial activities of the pyramidal cells $[x(0, \theta)]$ were taken from a uniform distribution of $[00.05]$ in all the simulations. Simulations were performed by MATLAB (Mathworks). Specifically, ordinal differential equations were numerically solved by using the "ode 45 " function that uses a varying time step, except for those considering dynamic noise (see Fig. 14), which were solved by the fourthorder Runge-Kutta method with a fixed time step of 0.1 [I have also done some simulations with static noise by using the Runge-Kutta method instead of ode45, confirming that the results looked similar (data not shown)]. Unless otherwise described, simulations were repeated for 50 times (for Fig. 14) or 100 times (for all the others) with the same condition except for the presumed randomness (initial activity of the cells and static or dynamic noise in the input) so as to calculate the probability of memory formation as well as the memory accuracy (for the definition of those terms, see Results).

Dependence on the parameters. In most simulations, the strength of recurrent excitation was fixed, whereas the magnitude of feedforward input and the strength of somatic and/or dendritic recurrent inhibitions were systematically varied. Therefore, in effect, relative magnitudes between those different kinds of inputs/connections were systematically varied, whereas the relationship between them (connection strengths) and the dendritic threshold and saturation was not. (Note that the model is otherwise scalable; especially, the model is scalable except for the dendritic saturation in the moderately nonlinear dendrite case.) I have, however, performed simulations with different strength of recurrent excitation (33\% increased or $20 \%$ decreased) under several (although not all) configurations regarding the type of dendritic nonlinearity and recurrent inhibition, and confirmed that the circuit behavior that I propose as a feature of the model incorporating dendritic compartmentalization still 



Figure 1. Schematic diagram of the model architecture. $\boldsymbol{A}$, Excitatory pyramidal cells (bottom pyramids) receive input ["(external/feedforward) input to the memory circuit"] from neurons in the input layer (top circles) via the feedforward excitatory connections (red arrows). These feedforward connections are assumed to be topographically organized in a functional sense, but not necessarily in a physical sense, such that a pyramidal cell and an input layer neuron with similar location/angle preference (or memory/response field: black small arrows in the top circles and the bottom pyramids) are strongly connected (as indicated by the width of the red arrows). This feedforward excitation comprises the following two parts: axons of the input layer neurons (yellow-backed region) and dendrites of the pyramidal cells (green-backed region), between which there are feedforward synapses (small black-filled circles). $\boldsymbol{B}$, The pyramidal cells are connected by recurrent excitatory connections (red arrows), which are assumed to be organized such that the strength of synaptic coupling between two pyramidal cells decreases with the difference in their preferred angles (represented by the width of the red arrows). This recurrent excitation is assumed to be added to each dendritic branch, rather than to the soma, of the pyramidal cells, as illustrated schematically. C, The soma-targeting inhibitory interneurons (bottom left ellipse) are activated by the pooled activity of all the pyramidal cells; in turn, these interneurons give inhibition onto the somata of the pyramidal cells [inhibitory interneurons are not explicitly modeled but instead represented by negative signs preceding the connection strengths (see Materials and Methods)]. D, Similarly, the dendrite-targeting inhibitory interneurons (bottom right ellipse) are activated by the pooled activity of all the pyramidal cells (global inhibition) or of nearby cells (local inhibition) and these interneurons give inhibition onto individual dendritic branch of the pyramidal cells. Although soma-targeting and dendrite-targeting interneurons are schematically illustrated as separate cell populations in those figures, it might be more likely that a single cell population could implement both of them (for details, see Results, On the nature of recurrent inhibition; and Materials and Methods, Network architecture). $\boldsymbol{E}$, The neural circuit for spatial working memory was assumed to consist of topographically organized feedforward excitatory connections from neurons in the preceding visual cortices (top circles) to the pyramidal cells in the memory circuit (bottom pyramid), recurrent excitation between pyramidal cells, and either somatically or dendritically mediated recurrent inhibition. Nonlinear input integration was assumed to be imposed on each dendritic branch of the pyramidal cells (black square; see $\boldsymbol{H}$ ). For more details, see Materials and Methods. $\boldsymbol{F}$, Schematic view of the assumption regarding the distribution of the feedforward excitatory inputs onto the dendritic branches of pyramidal cells. The external space was assumed to be mapped, not only onto a population of neurons constituting the circuit ("topographic organization of the feedforward inputs"; left), but also onto a dendritic tree of individual component neurons (right), by virtue of the dendritic branch-specific plasticity rules (for details, see Results and Materials and Methods). The morphology of the pyramidal cell was taken from a layer 2/3 neocortical pyramidal cell model (Mainen et al., 1995) in the NEURON computational neuroscience appeared in such conditions (inhibition strength should also be changed, although there are certain margins in the excitation-inhibition ratio) (see Results). Examples under a particular configuration are shown in Figure 13, E and $F$. As shown in the figures and explained in the corresponding text, increasing recurrent excitation, which corresponds to decreasing the dendritic threshold relatively, resulted in better performance in terms of our criteria (explained in Results), whereas decreasing recurrent excitation led to the opposite result. Such a tendency was also observed in simulations under other configurations.

Possible extension of the model and the relationship to a previous model. The model of the present study described above is related to a model considered in a previous work by Morita et al. (2007), which studied how the behavior of the competitive neural network (Amari and Arbib, 1977; Fukai and Tanaka, 1997; Hahnloser et al., 2000; Xie et al., 2002) changes when multiple dendritic branches with independent nonlinearity are incorporated. The most prominent

model archive. $\mathbf{G}$, The number of input layer neurons and that of dendritic branches of every pyramidal cell are assumed to be the same, for simplicity, and each single dendritic branch of a given pyramidal cell receives feedforward input from a different single input layer neuron. The feedforward excitatory connection strength between each dendritic branch and the input layer neuron connected to the branch is assumed to have a bell-shaped distribution (indicated by the orange line) peaked at the input layer neuron with the same angle preference as the pyramidal cell ( $0^{\circ}$ in this figure). Note that, because of this assumption, dendritic branches connected to the input layer neurons with the angle preferences of $-45 \sim 45^{\circ}$ receive almost all the feedforward inputs, whereas other branches mainly receive recurrent inputs. In most of the simulations, recurrent excitation was assumed to be uniformly distributed over the branches (red solid line), although nonuniformly distributed recurrent excitation (red dashed line) was also examined in some simulations (Fig. 13D-F and the corresponding text). Note that the relative strength of the feedforward and recurrent excitation shown in the graph does not represent the actual value (for the values, see Materials and Methods). $\mathbf{H a}$, The nonlinearity of the input integration in the dendritic branches was modeled as a piecewise linear function, characterized by threshold, slope, and upper bound (saturation). I considered two different cases as follows. $\boldsymbol{H b}$, The case of moderately nonlinear dendrite. This could represent the situation of a short passive dendritic branch, in which input integration is nearly linear except for the saturation and branch restrictiveness of shunting inhibition, which was modeled by rectification (threshold at 0 ). The effect of inhibition applied onto the branch, when it was considered, was modeled as a subtraction (blue arrow). $\mathbf{H c}$, The case of highly nonlinear dendrite. This could represent the situation of a long active dendritic branch, in which the branch can contribute to somatic firing almost only when the excitation applied onto the branch exceeds a certain amount (represented as a positive threshold in the function) so that dendritic spikes are generated. The effect of inhibition applied onto the branch was modeled as a division on the input, resulting in a combination of division and subtraction on the output firing rate, as indicated by the reduction in the slope and the rightward shift (blue arrows), respectively. 
difference between the previous study and the present one is that the previous model (Morita et al., 2007) included only self-excitation of neurons, whereas the model in this study includes mutual (recurrent) excitation between pyramidal cells, which plays an essential role in the formation of a sustained bump of activity that represents a spatial memory. It might be possible to regard the previous model (Morita et al., 2007) as a model of object working memory, although that model was much simpler than the model in this study so that direct correspondence to biological circuits would be more difficult. However, taking advantage of the simplicity, we could perform mathematical analyses in the previous model by theoretically considering the limit at which every neuron has an infinite number of dendritic branches. In fact, mathematical analysis could also be done for the model of this study. If there are a large number of pyramidal cells, they can be represented as a "neural field" (Amari, 1977), in which each cell is continuously parameterized by the preferred angle $(\theta)$. Thus, the above-mentioned set of ordinary differential equations can be represented as an integro-partial-differential equation as follows:

$$
\frac{\partial x(t, \theta)}{\partial t}=-x(t, \theta)+\sigma_{s}\left(\sum_{\varphi} \sigma_{d}(J(\varphi, \theta))-a_{s} S(t)\right),
$$

where

$$
S(t) \equiv \int_{\theta^{\prime}} x\left(t, \theta^{\prime}\right) d \theta^{\prime}
$$

Likewise, assuming a large number of dendritic branches per pyramidal cell, the branches can be represented as a "dendritic field" in which each branch is continuously parameterized by the preferred angle of the input layer neuron $(\varphi)$, from which it receives feedforward excitation. Consequently, the equation can be further modified as follows:

$$
\frac{\partial x(t, \theta)}{\partial t}=-x(t, \theta)+\sigma_{s}\left(\int_{\varphi} \sigma_{d}(J(\varphi, \theta)) d \varphi-a_{s} S(t)\right),
$$

where

$$
J(\varphi, \theta)=F(\varphi, \theta) I\left(\varphi ; \varphi_{0}\right)+\int_{\theta^{\prime}} E\left(\theta, \theta^{\prime}\right) x\left(t, \theta^{\prime}\right) d \theta^{\prime}-H(t)
$$

(subtractive inhibition)

or

$$
J(\varphi, \theta)=\frac{F(\varphi, \theta) I\left(\varphi ; \varphi_{0}\right)+\int_{\theta^{\prime}} E\left(\theta, \theta^{\prime}\right) x\left(t, \theta^{\prime}\right) d \theta^{\prime}}{1+H(t)}
$$

(divisive inhibition),

which can be referred to as "neuro-dendritic field equation" (Morita and Amari, 2007) and could be further analyzed mathematically.

\section{Results \\ Modeling spatial working memory circuit incorporating pyramidal dendritic compartmentalization}

I simulated a spatial working memory task such that a visual stimulus (visual cue) was transiently presented at an arbitrary location on a circle, and the animal was expected to memorize the location or "angle" of the stimulus (Funahashi et al., 1989) (see Fig. 2A). It has been hypothesized that there is a neural circuit underlying such spatial working memory in the neocortex, which consists of topographically organized feedforward excitatory connections from sensory (visual) cortices to the principal (pyramidal) cells, recurrent excitation between pyramidal cells with the strength correlating with the similarity in the spatial selectivity (i.e., location/angle preference, or memory/response field), and recurrent inhibition via interneurons (Goldman-Rakic, 1995). Assuming such a basic network architecture, many computational models have been developed to explore cellular mechanisms of spatial working memory (Constantinidis and Wang, 2004). I constructed a model based on a similar network architecture, but incorporated multiple dendritic branches of the individual pyramidal cells (Fig. $1 E$ ) (see below) (for details, see also Materials and Methods). Pyramidal cellular activity was described as a scalar variable representing the firing rate, which was driven by the inputs on the dendritic branches as well as on the soma. Inhibitory interneurons were not explicitly modeled but instead represented by negative signs of the connection strength.

To incorporate multiple dendritic branches, distributions of various types of inputs over the branches should be determined. Recent experiments in the hippocampus have revealed that synaptic potentiation could be induced in the pyramidal cells by locally generated dendritic spikes coupled with presynaptic cellular firings, even without postsynaptic somatic action potential generation (Golding et al., 2002). Moreover, electrical (Mel, 1993) and biochemical (Govindarajan et al., 2006) cooperativity within individual dendritic branches was proposed (Poirazi and Mel, 2001; Mehta, 2004; Govindarajan et al., 2006), and actually demonstrated in the hippocampal CA1 pyramidal cells (Harvey and Svoboda, 2007), to define the dendritic branch as a functional unit of synaptic plasticity. It was also shown that the excitability of the dendrite can be changed and stored via activitydependent regulation of potassium channels in a branch-specific manner in the CA1 pyramidal cells (Losonczy et al., 2008). Although there has been no evidence for or against that similar rules can also be applied to other brain areas such as the neocortex and it should be noted that things could potentially differ (Matsuzaki, 2007), I assumed that such dendritic branch-specific plasticity rules operate in the spatial working memory circuit so that the external space is mapped, not only onto a population of neurons constituting the circuit (Fig. $1 F$, left) but also onto a dendritic tree of individual component neurons (Fig. $1 F$, right), in analogy with what has been proposed for visual cortical neurons (Mel et al., 1998; Archie and Mel, 2000). Specifically, I assumed that different dendritic branches of a given pyramidal cell receive feedforward inputs from different neurons in the preceding area (referred to as the input layer neurons) (Fig. 1G) (for details, see Materials and Methods). In addition, I assumed that such an intradendritic self-organization (Fig. $1 F$, right) occurs in parallel with the network level self-organization (Fig. $1 F$, left), namely, the topographical organization of the feedforward connectivity as considered in the existing models of spatial working memory, so that the connection strength between each dendritic branch of a given pyramidal cell and the input layer neuron has a bellshaped distribution peaked at the input layer neuron with the same angle preference as the pyramidal cell (Fig. $1 G$, orange line). With this assumption, most feedforward inputs are mapped onto some portion of the dendritic branches, whereas other branches mainly receive recurrent inputs (see below). If the same plasticity rule is applied to recurrent excitatory inputs, they would also be mapped onto the branches in a similar nonuniform manner. However, the situation is not the same as that of the feedforward inputs, because the pyramidal cells in the memory circuit are considered to fire randomly in the beginning of the circuit formation, contrary to the input layer neurons. Therefore, nonuni- 
formity of the distribution of the recurrent excitatory inputs, if it exists, is expected to be shaped later and/or to less extent than that of the feedforward inputs. Considering that, in most simulations, I assumed that recurrent excitation is evenly applied onto all the dendritic branches (Fig. 1G, red solid line). I also performed several simulations with recurrent excitation that is nonuniformly distributed in a similar manner to the feedforward inputs, although to less extent (Fig. 1G, red dashed line) (for details, see Materials and Methods), confirming that the circuit feature of our interest (explained later) is mostly preserved (see Fig. 13D-F and the corresponding text). If the intradendritic distributions of the feedforward and recurrent excitation are quite different from those assumed ones, however, circuit behavior may differ (for additional discussion, see Materials and Methods). Looking into additional details, dendrites of pyramidal cells can usually be divided into two morphologically distinct parts, namely, apical and basal dendrites. However, because exactly how various types of inputs are distributed onto those two parts seems to remain elusive, I did not explicitly incorporate such a distinction into the model. Nevertheless, it has been generally considered that basal and proximal apical/oblique dendrites of pyramidal cells tend to receive inputs from nearby cortical cells, whereas distal apical/ tuft dendrites tend to receive long-range corticocortical as well as thalamocortical inputs (Spruston, 2008). If such a principle, supposedly determined by genetic factors, is applicable to the pyramidal cell in the spatial working memory circuit, feedforward inputs are expected to be mapped onto the distal apical dendrite, whereas recurrent excitation is expected to be applied onto the proximal/basal dendrites. The above-mentioned model assumption (Fig. $1 G$ ) might also be approximately in line with such an apical-basal/proximal distinction, with the branches receiving a considerable amount of feedforward inputs corresponding to the apical/distal dendrites and the rest representing the basal/proximal dendrites, although more precise modeling would be desired when detailed data become available in the future. Regarding recurrent inhibition, I considered a somatically mediated one (Fig. 1C) and a dendritically mediated one (Fig. 1D). Dendritically mediated recurrent inhibition, when it was considered, was assumed to be evenly applied onto all the dendritic branches (although inhibitory inputs can also be nonuniformly distributed in reality, exact distribution for recurrent inhibition is not known at the moment so that the uniform distribution was assumed). I initially assumed that recurrent inhibition, either somatic or dendritic, is recruited by the activity of all the pyramidal cells and, in turn, equally affects every pyramidal cell (i.e., operates as global recurrent inhibition). However, later I also examined the case in which dendritic inhibition is more localized (see Fig. 11A) (see Materials and Methods). Although soma-targeting and dendritetargeting interneurons are schematically illustrated as separate cell populations in Figure 1, $C$ and $D$, it might be more likely that a single-cell population could implement both of them (for details, see below, On the nature of recurrent inhibition, and above, Materials and Methods, Network architecture).

I modeled the nonlinearity of the input integration on each branch (Fig. $1 E$, black square) as a piecewise linear function, a mathematical formula characterized by threshold, slope, and upper bound (saturation) (Fig. $1 \mathrm{Ha}$ ). Two different cases were examined (Fig. $1 \mathrm{Hb}, \mathrm{Hc}$ ). First, if the dendritic branch is relatively passive and short, integration of the excitatory inputs is expected to be approximately linear except for the saturation attributable to shunting (Prescott and De Koninck, 2003), and voltage attenuation could be ignored. Moreover, inhibition applied onto a branch is expected to effectively shunt the excitatory inputs on the same branch but hardly affect the other branches because of the nature of shunting inhibition, as theoretically predicted (Koch et al., 1983; Shepherd and Brayton, 1987) and experimentally demonstrated (Liu, 2004). Therefore, the relationship between the input to the branch and the output current from the branch to the soma could be approximated by a threshold-linear function with a saturation (Fig. $1 \mathrm{Hb}$, solid line), in which the threshold at 0 (i.e., rectification) represents the branch restrictiveness of the shunting inhibition [how positive deviation of the threshold affects the circuit behavior will be considered later (see Fig. $10 A a, B$ ) (see Materials and Methods)]. The effect of inhibition on the somatic firing rate was described as a subtraction, based on a known result (Holt and Koch, 1997) (Fig. 1 Hb, blue arrow). Next, however, if the dendritic branch is much more active, local dendritic spikes are likely to be generated when the excitation onto the branch exceeds a certain level within certain temporal and spatial windows (Schiller et al., 2000; Wei et al., 2001; Polsky et al., 2004; Gasparini and Magee, 2006; Losonczy and Magee, 2006; Milojkovic et al., 2007; Nevian et al., 2007), resulting in supralinear input integration above the threshold level. Moreover, if the branch is relatively long, small EPSPs are attenuated on the way to the soma, and thereby the branch can affect somatic firing almost exclusively via generating dendritic spikes. The input-output relationship of such a highly active branch could therefore be better approximated by a function with a positive threshold, rather than the rectification (zero threshold), and a steep slope (Fig. $1 \mathrm{Hc}$, solid line). Random variation on the threshold, slope, and saturation will be considered later (see Fig. $10 A b, A c, C$ ) (see Materials and Methods). Inhibition applied onto such an active branch is again expected to have branch-specific effect [i.e., prevention of the dendritic spike generation on the same branch, as predicted by detailed numerical simulations (Rhodes, 2006)]. However, its effect on the somatic firing rate might not be described as a subtraction as considered above, but instead as a division on the excitatory input to the branch, which results in a combination of divisive and subtractive effect on the output firing rate, when inhibition is asynchronous (Jadi and Mel, 2007) (Fig. $1 \mathrm{Hc}$, blue arrows). I will refer to the above-mentioned two cases as moderately nonlinear dendrite (Fig. $1 \mathrm{Hb}$ ) and highly nonlinear dendrite (Fig. $1 \mathrm{Hc}$ ), respectively. It was suggested that the degree of nonlinearity of the dendritic integration would depend on the spatiotemporal property of the synaptic inputs (Gasparini and Magee, 2006); however, because individual synaptic inputs were not described in our model, considering temporal features would be beyond the scope of the current study (additional discussion on this matter, however, will be provided later). In both moderately and highly nonlinear dendrite cases, output current from all the branches were assumed to be linearly summated, and then somatically mediated recurrent inhibition was implemented as a subtraction. Finally, this resulting value was subject to a threshold nonlinearity representing action potential generation at the soma (i.e., the value was rectified to zero if it was negative).

I examined the behavior of the circuit model in response to a transiently presented visual stimulus (Fig. 2 A). Specifically, it was assumed that a visual stimulus was transiently presented at the angle $0^{\circ}$ (for simplicity), and the working memory circuit received a visually evoked feedforward input from preceding brain areas during this duration. I considered two distinct parameters that could characterize the feedforward input to the memory circuit from preceding visual regions, namely, input contrast (Fig. $2 \mathrm{Ba}$ ), which is the ratio of the stimulus-location (angle)correlated component to the background, and "input intensity" 


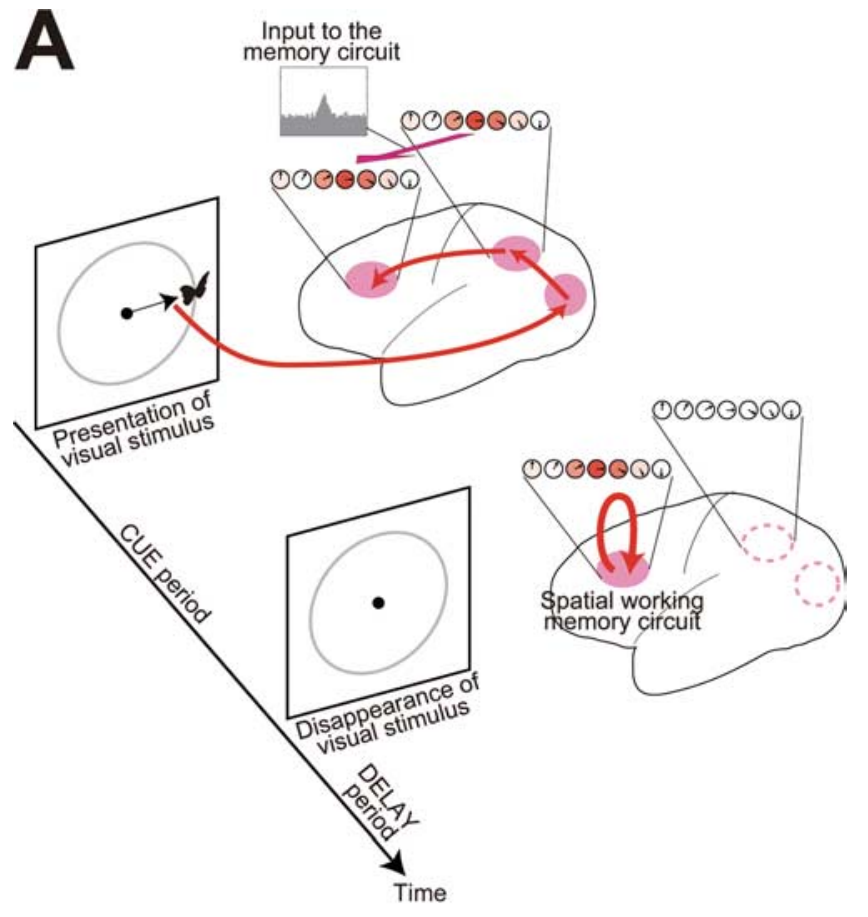

$\mathbf{B}_{\mathbf{a}}$


Figure 2. Experimental design of the simulation. $\boldsymbol{A}$, Schematic diagram of the simulated spatial working memory task. A visual stimulus (visual cue) was transiently presented at an arbitrary location on a circle, and the animal was expected to memorize the location or angle of the stimulus. I modeled the neural circuit for spatial working memory (located in the prefrontal cortex as illustrated in the figure or in other association cortices), which receives stimulus-evoked feedforward inputs including the information about the stimulus location (angle) from preceding visual cortices during the stimulus presentation (denoted as the CUE period). After the disappearance of the visual stimulus (denoted as the DELAY period), activities in the visual cortices diminish, whereas the memory circuit sustains activity via recurrent inputs. In the simulations, the visual stimulus was assumed to appear at the angle $0^{\circ}$ for the sake of simplicity. $\boldsymbol{B} \boldsymbol{a}, \boldsymbol{B} \boldsymbol{b}$, Two distinct parameters characterize the input to the memory circuit. $\mathbf{B} \boldsymbol{a}$, The input contrast (i.e., the ratio of the background to the stimulus angle-correlated component) is varied: 0 (left), 0.5 (middle), and 1.0 (right). $\boldsymbol{B} \boldsymbol{b}$, The input intensity is varied [i.e., both the background $\left(I_{0}\right)$ and the stimulus angle-correlated component $\left(I_{s}\right)$ are coordinately changed: $I_{0}=0.05$ (left), $I_{0}=$ 0.1 (middle), and $I_{0}=0.15$ (right)] while the input contrast is kept constant $\left(I_{\text {s } \max } / I_{0}=\right.$ 1.0). I examined the circuit behavior with systematically varying those two parameters, as well as the other assumptions such as the type of nonlinearity of the dendritic input integration (Fig. $1 \mathrm{H}$ ), or the type and the strength of recurrent inhibition (for details, see Materials and Methods).
(Fig. $2 \mathrm{Bb}$ ) (for details, see Materials and Methods). I examined the circuit behavior with systematically varying those two parameters under various conditions regarding the nature of dendritic nonlinearity and recurrent inhibition.

\section{Behavior of the circuit with moderately nonlinear dendritic branches and somatically mediated recurrent inhibition} First, I examined the behavior of the circuit with moderately nonlinear dendritic branches (Fig. $1 \mathrm{Hb}$ ) under the assumption that globally operated recurrent inhibition was mediated by soma-targeting interneurons. Figure $3 A$ shows the input to the memory circuit (panels in the top row), resulting temporal evolution of the pyramidal cellular activities (second row), and the activities at a time after stimulus extinction (i.e., in the "delay period") (bottom row). The results for three different input contrasts, 0.8 (Fig. 3Aa), $0.4(A b)$, and $0(A c)$, are shown. When the input contrast was high (Fig. $3 A a$ ), the pyramidal cells whose preferred angles were in proximity to the stimulus angle $\left(0^{\circ}\right)$ became highly active immediately after the appearance of the visual stimulus, whereas the other cells remained silent. This activity "bump" was sustained after the disappearance of the stimulus (Fig. 3Aa, bottom); thus, it can be considered to represent the "memory" of the stimulus location (angle). When the input contrast was decreased, the activity bump was still formed and sustained (Fig. 3Ab). However, there was greater trial-to-trail variation in the center of the bump, which corresponded to the "memorized angle," as shown for two trials in Figure $3 A b$. Even in the case in which the input contrast was 0 , thereby indicating that the input to the memory circuit did not include stimulus anglecorrelated component (Fig. 3Ac), the activity bump was still formed and sustained. However, the location of the bump was randomly determined, thereby indicating compulsive formation of spurious memory. Figure $3 B$ summarizes the results of many trials at four different input contrasts. Histograms (Fig. 3B, left) show the distributions of the center of the activity bump (i.e., memorized stimulus angle). When the input contrast was high (Fig. 3B, left, top), the distribution peaked sharply at the stimulus angle $\left(0^{\circ}\right)$, indicating accurate memory formation. The distribution continued to peak despite the decrease in the input contrast (Fig. 3B, left, second and third); however, the variation increased significantly. When the input contrast was 0 (Fig. 3B, left, bottom), the distribution was flat, thereby indicating again the formation of random spurious memory. The circles (Fig. 3B, right) show instances of the memorized angle (red lines: $<250$ trials are drawn) and its vector average (thick black line: averaged over all the trials), whose length is considered to indicate the accuracy with which the memory provides information regarding the stimulus angle on average; therefore, I refer to it as "accuracy." Figure $3 C$, solid line, indicates the accuracy. As shown in the figure, the accuracy decreased with decrease in the input contrast. I examined how such dependence of the memory accuracy on the input contrast changed when the intensity of the input to the memory circuit (Fig. $2 B b$ ) was varied. Figure $3 C$, dashed and dotted lines, indicates the accuracy of memory in the cases in which the input intensity was doubled or halved (i.e., the background and the stimulus angle-correlated component of the input to the memory circuit were simultaneously doubled or halved, respectively). As shown in the figure, the accuracy decreased along with the decrease in the input contrast in all cases. 
A

a

Contrast 0.8
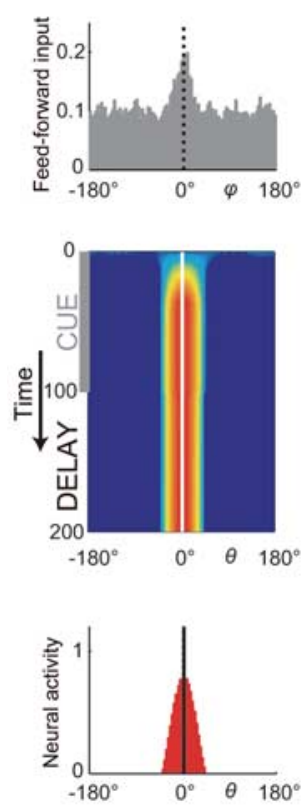

b



\section{Contrast 0.4}
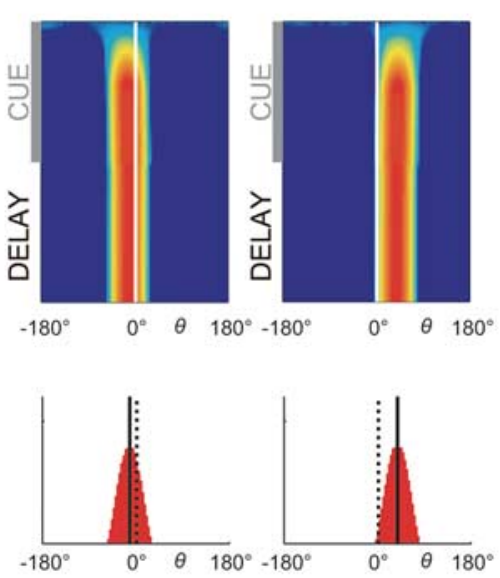

C

\section{Contrast 0}


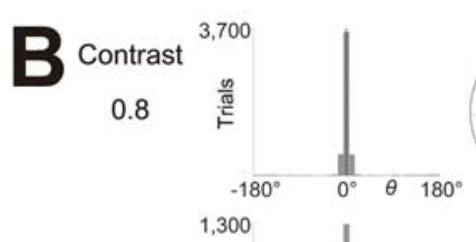

0.4
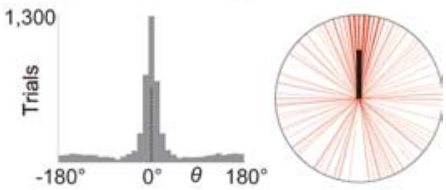

900


0
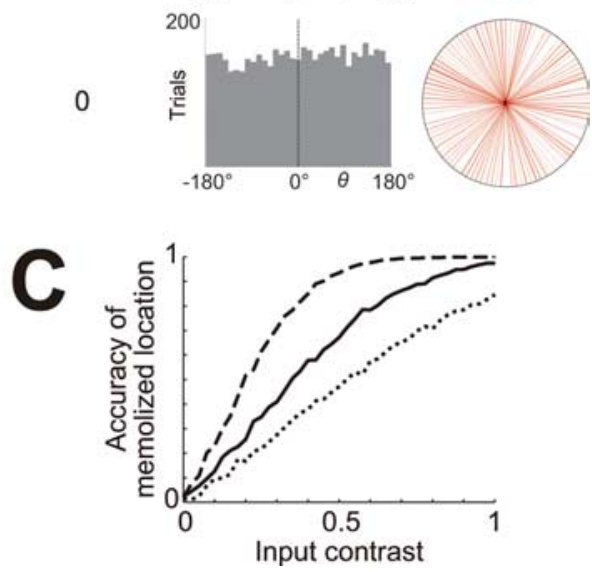

Figure 3. Behavior of the memory circuit with moderately nonlinear dendrites and somatically mediated recurrent inhibition. Circuit behavior was examined with systematically varying the contrast of the input to the memory circuit (Fig. $2 \mathrm{Ba}$ ). The input intensity (Fig. $2 \mathrm{Bb}$ ) was kept constant, except for in $\mathbf{C}$. $\boldsymbol{A a}-\boldsymbol{A c}$, Example trials for three different input contrasts are illustrated: 0.8 $(\boldsymbol{A} \boldsymbol{a}), 0.4$ (two trials are drawn) $(\boldsymbol{A} \boldsymbol{b})$, and $0(\boldsymbol{A c})$. Images in the second row represent the time evolutions of the pyramidal cellular activities. The horizontal axes indicate neuron indexes with preferred angles from $-180^{\circ}$ (left) to $180^{\circ}$ (right). The vertical axes indicate time (from top to bottom). The visual stimulus was assumed to be transiently presented, as indicated by vertical gray bars to the left of the images. The panels in the bottom row represent activities of the pyramidal cells in the DELAY period after stimulus extinction. The vertical black solid lines indicate the center of the activity bump, whereas black dotted lines indicate the stimulus angle $\left(0^{\circ}\right)$. When the input contrast was high $(\boldsymbol{A a})$, the activity bump was formed at the location (angle) of the visual stimulus, indicating accurate memory formation. As the input contrast decreased, the trial-to-trial variation in the activity bump (i.e., memorized angle) increased, as shown for two trials in $\boldsymbol{A b}$. When the input contrast was zero (c), activity bump was induced at a random location, indicating the formation of random spurious memory. $\boldsymbol{B}$, Summary of the results of many trials for four different input contrasts. The histograms on the left show distributions of the center of the activity bump (i.e., memorized angle) in the DELAY period (at time 200) for 5000 trials. When the input contrast was high (top), the distribution peaked sharply at the stimulus angle $\left(0^{\circ}\right)$, indicating accurate memory formation. The distribution continued to peak despite the decrease in the input contrast (second and third panels); however, the variation increased significantly. When the input contrast was 0 (bottom), the distribution was flat, indicating the formation of random spurious memory. The circles on the right show examples of the memorized angle (thin red lines: 250 trials are drawn) and its vector average (thick black line: averaged over all 5000 trials), whose length is considered to represent how accurately the memory provided information regarding the stimulus angle on average, and thus referred to as the accuracy. $C$, Dependence of the accuracy of spatial memory on the input contrast. The solid line indicates a case with the original parameter used in the simulation in $\boldsymbol{A}$ and $\boldsymbol{B}$, whereas the dashed and dotted lines indicate cases in which the input intensity (Fig. $2 B b$ ) was doubled or halved, respectively. The accuracy decreased with decrease in the input contrast in all cases.

\section{Behavior of the circuit with moderately nonlinear dendritic branches and dendritically mediated recurrent inhibition}

Next, I examined the case in which recurrent inhibition operated again globally, but was mediated by dendrite-targeting interneurons rather than by soma-targeting cells. Figure 4 shows the results. When the input contrast was high (Fig. $4 A a$ ), the circuit behavior was similar to that observed for somatically mediated recurrent inhibition (Fig. 3Aa) (i.e., the activity bump was formed and sustained after stimulus extinction). However, as the input contrast decreased (Fig. $4 A b$ ), the circuit behavior varied from the somatic inhibition case (Fig. $3 A b$ ). The activity bump was formed and sustained in some trials (Fig. 4Ab, trial 1). However, in others (trial 2), the pyramidal cells shared small activities (indicated by light blue color in the second panel) during the stimulus presentation, and the activity was not sustained after stimulus extinction. When the input contrast was zero, the activity bump was never formed nor sustained (Fig. 4Ac). The prob- ability of the formation and sustenance of the activity bump (i.e., the probability of memory formation) decreased as the input contrast decreased, as appeared in the red line in Figure 4C. More precisely, the probability decreased substantially around a certain level of input contrast, thereby suggesting the existence of a "threshold contrast" for memory formation [the curve becomes even steeper when the input has time-varying noisy component (dynamic noise) as we shall see later (see Fig. 14)]. I examined how such a dependence of the memory formation on the input contrast changed when the input intensity was varied (Fig. $2 B b$ ). Figure $4 C$, black dashed and dotted lines, indicates the probability of memory formation in the cases in which the input intensity was doubled or halved, respectively. As shown in the figure, these lines are so close to the red line (result for the original input intensity) that they are almost hidden, indicating that the contrast dependence of the memory formation was hardly affected by the change in the input intensity. In other words, memory for- 
A

a

\section{Contrast 0.8}
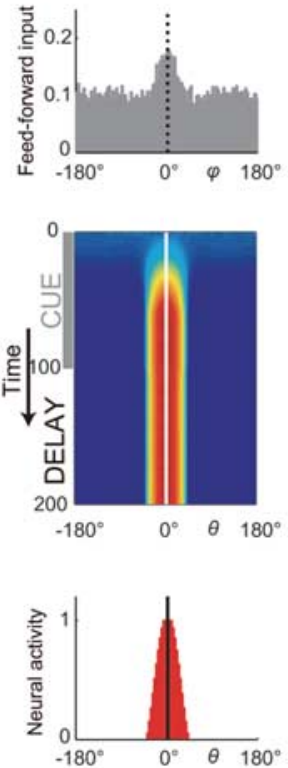

b

\section{Contrast 0.4}

trial 1
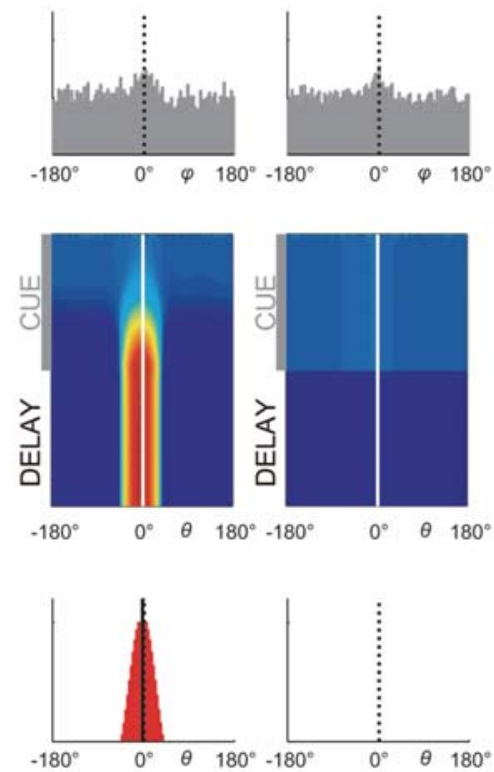

C

\section{Contrast 0}
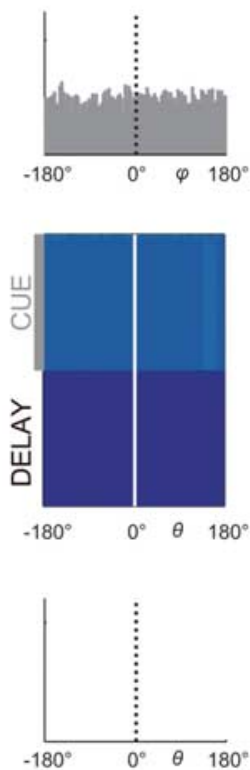

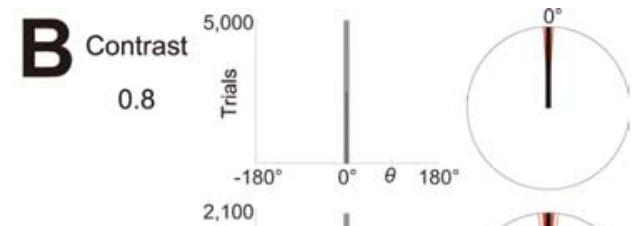

0.4



0.3


D

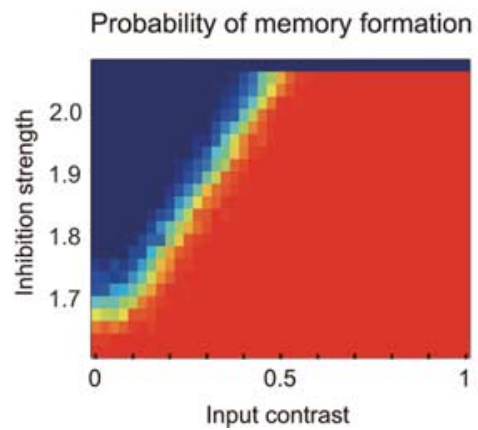

Accuracy of memolized location
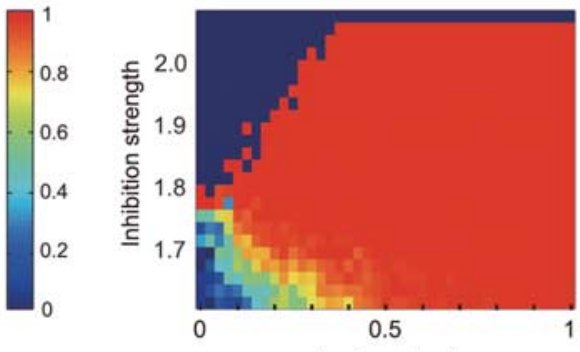

Input contrast

Figure 4. Behavior of the memory circuit with moderately nonlinear dendrites and dendritically mediated recurrent inhibition. The sametask as in Figure 3 was tested for the case with dendritically mediated recurrent inhibition. $\boldsymbol{A a}-\mathbf{A c}$, The configurations are the same as in Figure $3 A$. When the input contrast was high $(\boldsymbol{A a})$, activity bump was formed at the stimulus location and sustained after stimulus extinction, just similar to the case with somatically mediated recurrent inhibition (Fig. 3Aa). As the input contrast decreased ( $\boldsymbol{A} \boldsymbol{b}$ ), the activity bump was formed and sustained in some trials (trial 1). However, in other trials (trial 2), the pyramidal cells shared small activities (indicated by light blue color in the second panel) during the stimulus presentation, and the activity was not sustained after stimulus extinction. When the input contrast waszero (Ac), the activity bump was never formed nor sustained. $\boldsymbol{B}$, Summary of the results of many trials for four different input contrasts. The configurations are the same as in Figure $3 B$. The histograms (left) and the vector averages of the memorized angle (thick black lines in the right circles) were calculated for all the trials in which the bump was formed, of a total of 5000 trials. As shown in the histograms, the variation in the memorized angle remained small even when the input contrast was low, in contrast to the case with somatic inhibition (Fig. 3B). At input contrast of 0 (bottom), no bump activity was observed; thus, the histogram is blank. C, The red line indicates the probability of the formation of the activity bump, i.e., memory formation, depending on the input contrast (horizontal axis). The cases with doubled and halved input intensities are indicated by the black dashed and dotted lines, respectively, although they are so close to the red line that they are almost hidden. As shown here, the probability of memory formation decreased as the input contrast decreased, and such contrast dependence was hardly affected by the change in the input intensity (intensity invariant at least in a certain range). The black solid line indicates the accuracy of spatial memory (plotted only for the input contrast with which memory could be formed), defined as the length of the averaged vector of memorized angles ( $\boldsymbol{B}$, thick black lines in the right circles). As shown here, memory was accurate whenever it was formed. Spurious memory was never formed, unlike that observed with somatic inhibition. $D$, Dependences of the probability of memory formation (left) and the accuracy of memorized location (right) on the input contrast (horizontal axes) for various inhibition strength (vertical axes). For a range of inhibition strength (between 1.85 and 2.05 ), memory was formed only when the input contrast was higher than a certain level (left) and the memory was accurate whenever it was formed (right) (i.e., the circuit was capable of the contrast-dependent accurate memory formation).

mation was shown to be almost solely contrast dependent, and intensity invariant at least in a certain range. Figure $4 B$ shows the distributions of the memorized angle over many trials at four different input contrasts. As shown in the figures, there was little variation in the memorized angle between the trials. Indeed, the variation was substantially smaller than that observed with somatic inhibition (Fig. 3B), even when the input contrast was so low that the probability of memory formation was quite low (Fig. 
A
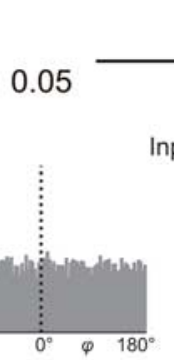

Input contrast

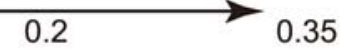

a

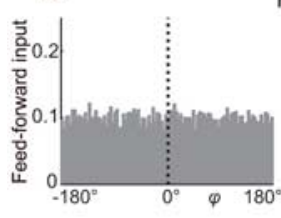

nput to the memory circuit


b

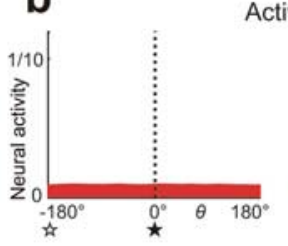

Activities of the pyramidal cells
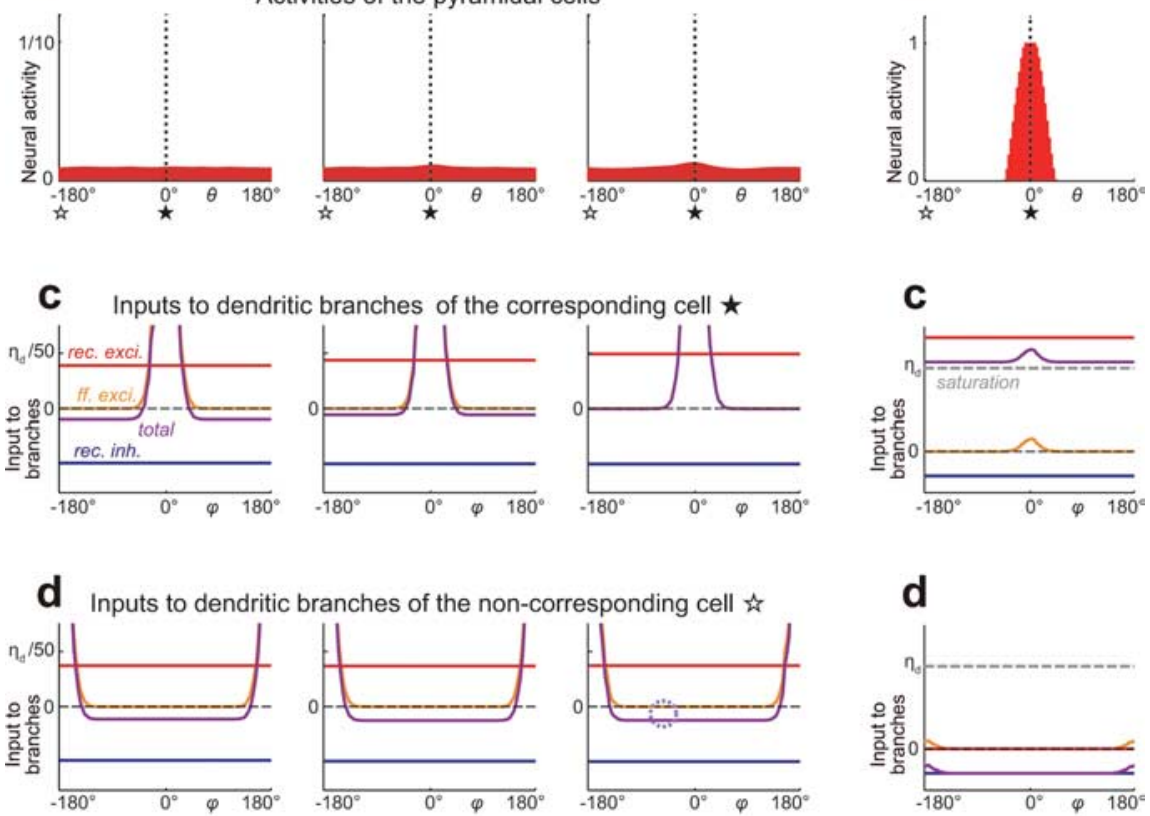

b



C

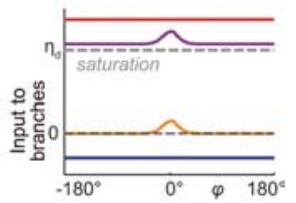

a

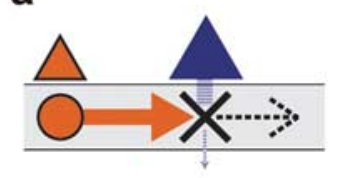

b

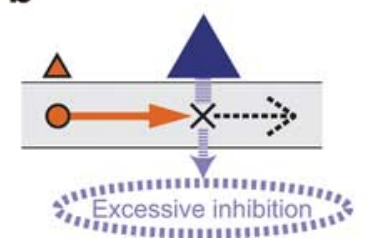
ulations, is instead set to a positive value. However, in such a case, contrastdependent memory formation will occur only for a limited range of input intensity; if the input intensity is near the value of the positive somatic threshold, memory is expected to be induced for a high-contrast input simply because it exceeds the threshold. To realize contrast-dependent mem-

\section{$\leftarrow$}

inhibition become equal (i.e., the inhibition is just necessary and sufficient for nullifying the excitatory effect) (Ac, right: the purple line is around 0 in most parts) (see also $(a)$. If the input contrast increases any further, the total input will become positive in every branch of the corresponding cell $(\boldsymbol{B})$. Subsequently, the cellular activity will increase significantly, thereby causing positive feedback via the recurrent excitation between the adjacent cells and result in memory formation. Therefore, this input contrast $(\sim 0.35)$ is considered to be a critical value (threshold contrast for memory formation that in fact well matches the simulation results shown in Fig. 4C). Note that if the input intensity is varied, everything except for the saturation is scaled accordingly, and thereby the above-mentioned mechanism of the contrast-dependent memory formation continues to hold. The light purple dashed circle in the right panel of Ad indicates prominent "excessive inhibition" (see (b) for the noncorresponding cell, whereas there is little such a waste for the corresponding cell as shown in Ac. B, Pyramidal cellular activities and the magnitude of each component of the inputs to the dendritic branches while the circuit was receiving the high contrast input ( 0.5$)$ that induced activity bump. The configurations are the same as in $\mathbf{A}$. $\mathbf{C a}, \boldsymbol{C b}$, Schematic diagrams illustrating how inhibition applied to a dendritic branch works. Ca, Inhibition is just as necessary and sufficient for nullifying the effect of excitation that is applied to the same branch. $\boldsymbol{C} \boldsymbol{b}$, Inhibition is more than sufficient to nullify the excitation. 
ory formation for a wider range of input intensity, certain elaborative mechanisms such as finely tuned feedforward inhibition needs to be additionally incorporated. In contrast, as far as the dendritic nonlinearity is described as the zero-threshold-linear function with a saturation (Fig. $1 \mathrm{Hb}$ ), dendritically mediated global subtractive recurrent inhibition enabled the contrastdependent accurate memory formation for a wide range of input intensity without any additional mechanisms, as shown above.

I examined how the circuit behavior changed if the inhibition strength, or the balance between excitation and inhibition, was varied. Figure $4 D$ shows the dependences of the probability of memory formation (left panel) and the accuracy of memorized location (right panel) on the input contrast (horizontal axes) with varying the inhibition strength (vertical axes). As shown in the figure, for a range of inhibition strength (between 1.85 and 2.05), memory was formed only when the input contrast was higher than a certain threshold (left panel) and the memory was accurate whenever it was formed (right panel) (i.e., the circuit was capable of contrast-dependent accurate memory formation). If inhibition was much weaker, memory was induced more easily but it was no longer accurate, as indicated by yellow or light blue colors in the bottom region of Figure $4 D$, right panel. In other words, the circuit behavior became more similar to that in the case of somatically mediated recurrent inhibition. In contrast, if inhibition was much stronger, memory could not be induced regardless of the input contrast, as indicated by the blue color in the topmost region of Figure $4 D$, left panel. Note that this also occurred in the case with somatically mediated recurrent inhibition (data not shown).

\section{Mechanism of contrast-dependent accurate memory formation via dendritic inhibition}

Let us explore the origin of such contrast-dependent accurate memory formation by dendritically mediated recurrent inhibition. As shown in the simulation (Fig. 4C), when the input contrast was lower than a certain threshold (Fig. $4 C, \sim 0.35$ ), pyramidal cells never developed an activity bump but, instead, shared almost uniform low activities (Fig. 5Aa,Ab). What combination of different components of input to the pyramidal dendritic branches can stabilize such a low activity state? Figure 5, Ac and $A d$, shows the magnitude of each component of inputs to the dendritic branches [parameterized by the preferred angle $(\varphi)$ of the input layer neuron that is connected to the branch (Fig. 1G)] of the pyramidal cell whose preferred angle matches the stimulus angle $\left(0^{\circ}\right)$, which is referred to as the "corresponding cell" (Fig. $5 A b$, filled star, $A c)$, and the cell whose preferred angle is the opposite of the stimulus angle $\left(-180^{\circ}\right)$, which is referred to as the "noncorresponding cell" (Fig. 5Ab, open star, $A d$ ), while the circuit is receiving the input with three different contrasts (three columns). Feedforward excitation (Fig. $5 A c, A d$, orange lines), recurrent excitation (red), dendritically mediated recurrent inhibition (blue), and the summation of those three (purple) are drawn. When the input contrast is very low (0.05) (Fig. $5 A$, left column), in most branches except those in proximity to the preferred angle of the cell $\left[0^{\circ}(c)\right.$ or $\left.-180^{\circ}(d)\right]$, the contribution of feedforward excitation is little. Recurrent excitation and inhibition are mostly balanced; however, the inhibition is slightly dominant; therefore, the result of summation (purple lines) is negative, even for the corresponding cell (Fig. 5Ac). Thus, the "output" of those branches is 0 because of the presumed dendritic threshold at 0 (Fig. $1 \mathrm{Hb}$ ). In the results, the soma collects positive current only from a small portion of the branches that receive considerable amount of feedforward excitation; thus, the cellular activity remains small, never reaching a level that can be sustained after stimulus extinction. As the input contrast increases (Fig. 5A, left to middle), the feedforward as well as the recurrent excitation that the corresponding cell receives increases accordingly (Fig. $5 A c$, orange and red lines, respectively). Consequently, the total input increases but it still remains negative for most branches (Fig. 5Ac, middle, purple line); thus, their dendritic outputs remain 0 , thereby preventing memory formation. As the input contrast increases more (Fig. $5 \mathrm{Ac}$, right), at a certain point, for most branches of the corresponding cell, the magnitudes of excitation and inhibition become equal (i.e., the inhibition is just necessary and sufficient for nullifying the excitatory effect, as indicated by a total input of 0 ) (Fig. $5 A c$, right, the purple line is around 0 in most parts). If the input contrast increases any further, the total input will become positive in every branch of the corresponding cell (Fig. 5B). Subsequently, the cellular activity will increase significantly, thereby causing positive feedback via the recurrent excitation between the adjacent cells and resulting in memory formation. Therefore, the input contrast in the case of the right panels of Figure $5 A(0.35)$ is considered to be approximately a critical value, which in fact well matches the threshold contrast for memory formation observed in the simulation (Fig. 4C). Note that, when the memory is formed (Fig. $5 B$ ), every dendritic branch of the corresponding cell, whose location preference perfectly matches that of the stimulus by definition and whose activity is at the maximum of the bump (Fig. $5 B b$ ), is at the saturated level (Fig. $5 B C$ ) that would represent successive or prolonged dendritic spike generation, whereas the branches of the cell whose preference is less similar to the stimulus (on the left and right slopes of the activity bump in Fig. $5 B b$ ) receive suprathreshold inputs but are not necessarily saturated.

Now let us explore another way of explanation. When the input contrast is low, inhibition is more than sufficient for completely inactivating the branch in most dendritic branches (Fig. $5 A c, A d)$. Because of the presumed branch specificity of the dendritic inhibition, such "excessive" inhibition cannot be used for other more active branches, but just almost "wasted" (Fig. 5C). Because inhibition dominance is more prominent in the noncorresponding cell than the corresponding cell (Fig. $5 \mathrm{Ad}$, right, light purple dashed circle), such a waste occurs more significantly in the noncorresponding cell, and subsequently, the "effective" amount of inhibition is greater in the corresponding cell. This is a form of negative feedback, which is especially prominent when the input contrast is just below the threshold for memory formation, or conversely, which defines such a threshold.

Why does such a contrast-dependent circuit behavior occur over a wide range of input intensity (Fig. 4C, dashed and dotted lines)? If the input intensity is varied (i.e., both the background and the stimulus angle-correlated components of the feedforward excitation are coordinately changed) (Fig. $2 \mathrm{Bb}$ ), recurrent excitation and recurrent inhibition applied onto dendritic branches should also be scaled accordingly, except for the effect of dendritic saturation. Therefore, the relative ratios between any input components on each dendritic branch are kept constant so that the above-mentioned mechanism of the contrast-dependent memory formation continues to hold, realizing the input intensity semiinvariance.

\section{Behavior of the circuit with highly nonlinear dendritic branches}

So far, I have assumed that the nonlinearity of the input integration on the dendritic branches was moderately nonlinear (Fig. $1 \mathrm{Hb}$ ). Next, I examined the cases in which the dendritic nonlin- 


\section{Somatically mediated recurrent inhibition}

a


Intensity 0.2


b
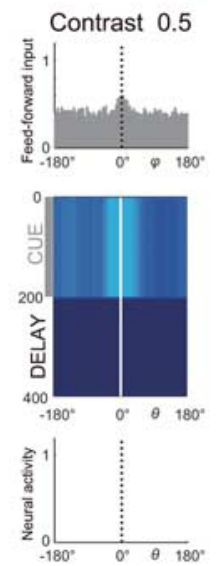

Intensity 0.4
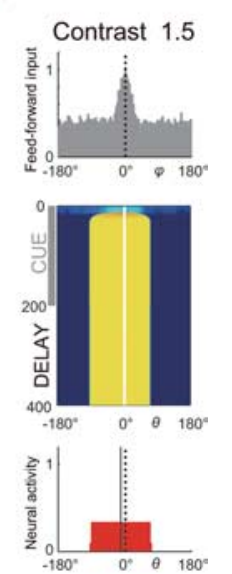

B

\section{Dendritically mediated recurrent inhibition}

a


Intensity 0.2
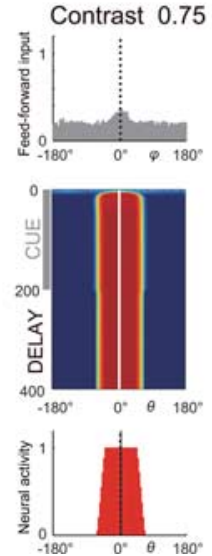

b


Intensity 0.4
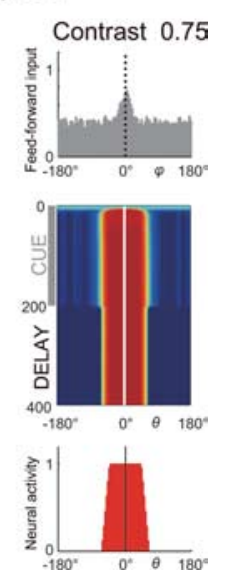

Figure 6. Behavior of the circuit with highly nonlinear dendritic branches. $\mathbf{A} \boldsymbol{a}-\boldsymbol{B} \boldsymbol{b}$, Results for the case with either somatically mediated subtractive recurrent inhibition $(\boldsymbol{A})$ or dendritically mediated divisive inhibition $(\boldsymbol{B})$ for two different input intensities $(\boldsymbol{a}$ and $\boldsymbol{b}$ ) are shown. The configurations are the same as in Figure $3 A$. With highly nonlinear dendrite, the circuit showed contrastdependent accurate memory formation for a range of input intensity not only when recurrent inhibition was mediated dendritically $(\boldsymbol{B})$ but also when it was mediated somatically $(\boldsymbol{A})$, in contrast to the previous cases with moderately nonlinear dendrite.

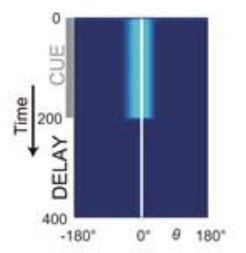

$7 A, B$, left columns), the total input activity is smaller than the dendritic threshold (indicated by light blue lines) in most branches except for those receiving significant feedforward excitation, even in the cell whose preferred angle is close to the stimulus location [corresponding cell $(c)$ ]. Consequently, those branches cannot contribute to somatic firing, preventing the formation of activity bump. It can be said, therefore, that the positive dendritic threshold plays a similar role to subtractive dendritic inhibition in the moderately nonlinear dendrite case in which the dendritic threshold was zero, realizing contrast-dependent accurate memory formation even if recurrent inhibition is mediated somatically (Fig. 5).

Because of the existence of the positive dendritic threshold, however, the circuit behavior should inevitably depend on the magnitude relationship between the threshold and the input intensity, contrary to the moderately nonlinear dendrite cases, and therefore the range of the input intensity for which contrast-dependent accurate memory formation holds would be limited. However, if recurrent inhibition is mediated dendritically, it is expected to partially compensate the change in the amount of feedforward excitation on each dendritic branch so that the contrast-dependent memory formation holds for a certain range of input intensity. Figure 8 shows the dependence of the circuit behavior on the inhibition strength. As shown in the figure, contrastdependent accurate memory formation was observed for a range of input intensity with either type of recurrent inhibition; the range was generally wider in the case with dendritically mediated recurrent inhibition (Fig. $8 \mathrm{~B}$ ), especially when the inhibition strength was finely tuned (strength 10: second panel from the left), earity was much more nonlinear (Fig. $1 \mathrm{Hc}$ ). Figure 6 shows the behavior of the circuit with such highly nonlinear branches, for the case with somatically mediated global subtractive recurrent inhibition (Fig. 6A) or with dendritically mediated (also global) divisive recurrent inhibition (Fig. $6 B$ ). As shown in the figure, the circuit showed contrast-dependent accurate memory formation not only when recurrent inhibition was mediated dendritically (Fig. 6B) but also when it was mediated somatically (Fig. 6A), contrary to the previously examined cases in which the dendritic nonlinearity was assumed to be moderate. Figure 7 shows individual components of the input to the pyramidal dendritic branches during the stimulus presentation for the case with somatically (Fig. 7A) or dendritically (Fig. 7B) mediated recurrent inhibition. The purple lines in the panels in $c$ and $d$ indicate the total input activity on the branches [i.e., summation of the feedforward excitation and the recurrent excitation (Fig. 7A) or that summation divided by dendritic inhibition (Fig. 7B)]. For both types of recurrent inhibition, when the input contrast is low (Fig. as expected above. When the inhibition strength was too weak, the width of the sustained activity bump became too wide (data not shown), and inaccurate memory could be induced (indicated by white crosses in the leftmost panel of Fig. $8 B$ ). In contrast, when the inhibition strength was too strong, memory became hard to be formed.

\section{On the number and heterogeneity of the dendritic compartments}

The number of independent electrical processing units, or "compartments," operating inside the neuron has been one of the major topics (Häusser and Mel, 2003). A study using a detailed model with real morphology of the pyramidal cell (Poirazi et al., 2003b) has indicated that several dozen long thin terminal branches comprise independent sigmoidal subunits. In the simulations I have so far shown, it was assumed that each pyramidal cell has 100 dendritic branches and each of those branches has its own nonlinearity (Fig. 1G). This value (100) seems to be in line 
with the above-mentioned study. However, the number of compartments could vary depending on the type of cells as well as on the state of synaptic inputs (Williams, 2004). To examine how the behavior of our circuit model depends on the number of compartments in individual pyramidal cells, I performed simulations with systematically varying the number of compartments. Specifically, I considered four cases. In all of them, the number of pyramidal dendritic branches, each of which receives feedforward input from an input layer neuron, were fixed to 135 . Thereafter, it was assumed that neighboring $1,5,15$, or 45 "terminal" branches were grouped into a single compartment, that is, all the excitatory inputs applied onto those $1,5,15$, or 45 branches were at first linearly summated and then inhibition, saturation, and thresholding were imposed, so that the pyramidal cell eventually had $135,27,9$, or 3 compartments for independent nonlinear input integration, respectively (Fig. 9A). The connection strength between a single terminal branch and the input layer neuron was assumed to have a bell-shaped distribution [Fig. $9 \mathrm{Ba}(\mathrm{i})]$ in the same manner as before (Fig. $1 G)$, and thus, in the case of total nine compartments [Fig. 9Ba(iii)], only three compartments received significant amount of feedforward excitatory inputs (one compartment receives most of them and the neighboring two receive the rest), or in the case of total three compartments [Fig. $9 \mathrm{Ba}(\mathrm{iv})$ ], only a single compartment received almost all the feedforward excitation.

Figure $9 B b$ shows how the circuit behavior changed depending on the number of compartments in the case with moderately nonlinear dendrite and dendritically mediated global recurrent inhibition. Probability of memory formation (left column) and accuracy of memorized location (right column) are plotted against the input contrast (horizontal axes) and the inhibition strength (vertical axes) for the cases with $135,27,9$, or 3 compartments [(i), (ii), (iii), and (iv), respectively]. As the number of compartments decreased [from (i) to (iv)], the threshold contrast for memory formation slightly decreased (left column) while the minimum amount of inhibition necessary for preventing the formation of inaccurate memory slightly increased (right column). However, the overall tendency did not primarily change, demonstrating that the main feature described above holds true even when the pyramidal cell has only a few "parallel" dendritic compartments for independent nonlinear input integration.

Figure $9 B c$ shows the highly nonlinear dendrite cases with either somatically mediated subtractive recurrent inhibition [(i)(iv), top rows] or dendritically mediated divisive recurrent inhi-
B Dendritically mediated inhibition
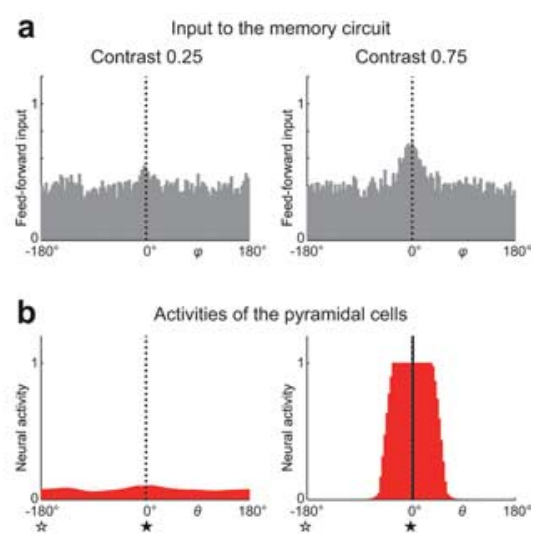

C Inputs to the branches of the corresponding cell $\star$



d Inputs to the branches of the non-corresponding cell \&

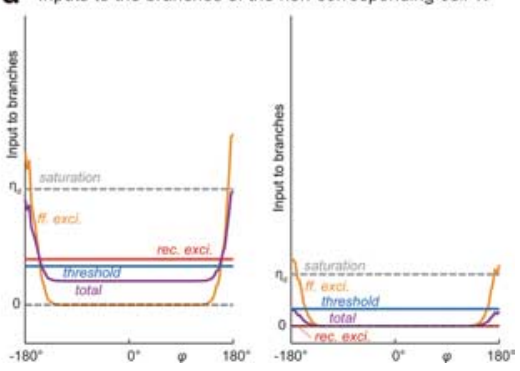

Figure 7. Mechanism underlying contrast-dependent memory formation in the cases of highly nonlinear dendrite. $\boldsymbol{A a}-\boldsymbol{B d}$, Individual components of the input to the pyramidal dendritic branches during the stimulus presentation for the case with somatically mediated subtractive recurrent inhibition $(\boldsymbol{A})$ or dendritically mediated divisive recurrent inhibition $(\boldsymbol{B})$. The configurations are almost the same as in Figure 5. $\boldsymbol{a}$, Input to the circuit. $\boldsymbol{b}$, Activity of the pyramidal cells (the filled stars indicate the corresponding cell whose preferred angle matches the stimulus, whereas the open stars indicate the noncorresponding cell having the opposite angle preference). $\boldsymbol{c}, \boldsymbol{d}$, Each component of the inputs to the dendritic branches of the corresponding cell (c) and of the noncorresponding cell (d), respectively. Feedforward excitation (orange lines), recurrent excitation (red lines), the dendritic threshold (light blue lines), and the dendritic saturation (gray dashed lines) are shown. The purple lines indicate the total input activity on the branches, that is, summation of the feedforward excitation and the recurrent excitation in $\boldsymbol{A}$, or that sum divided by dendritic inhibition in $\boldsymbol{B}$. For both types of recurrent inhibition, when the input contrast is low (left columns), the total input activity (purple line) is smaller than the dendritic threshold (light blue line), which was assumed to be positive (Fig. 1 Hc), in most branches except for those receiving significant feedforward excitation, even in the corresponding cell (c). Consequently, those branches cannot contribute to somatic firing, preventing the formation of inaccurate memory.

bition (bottom rows). Color indicates the probability of memory formation depending on the input contrast (horizontal axes) and the input intensity (vertical axes: log scale) with varying the inhibition strength (five columns), and white crosses indicate the formation of inaccurate memory (Fig. 8). As the number of compartments decreased from 135 to 27 [(i) to (ii)], the circuit behavior did not so much change. The circuit was still capable of contrast-dependent accurate memory formation for a range of input intensity; over twice difference for the case of somatically mediated inhibition (top rows) and up to eight times difference for the case of dendritically mediated inhibition (bottom rows). 


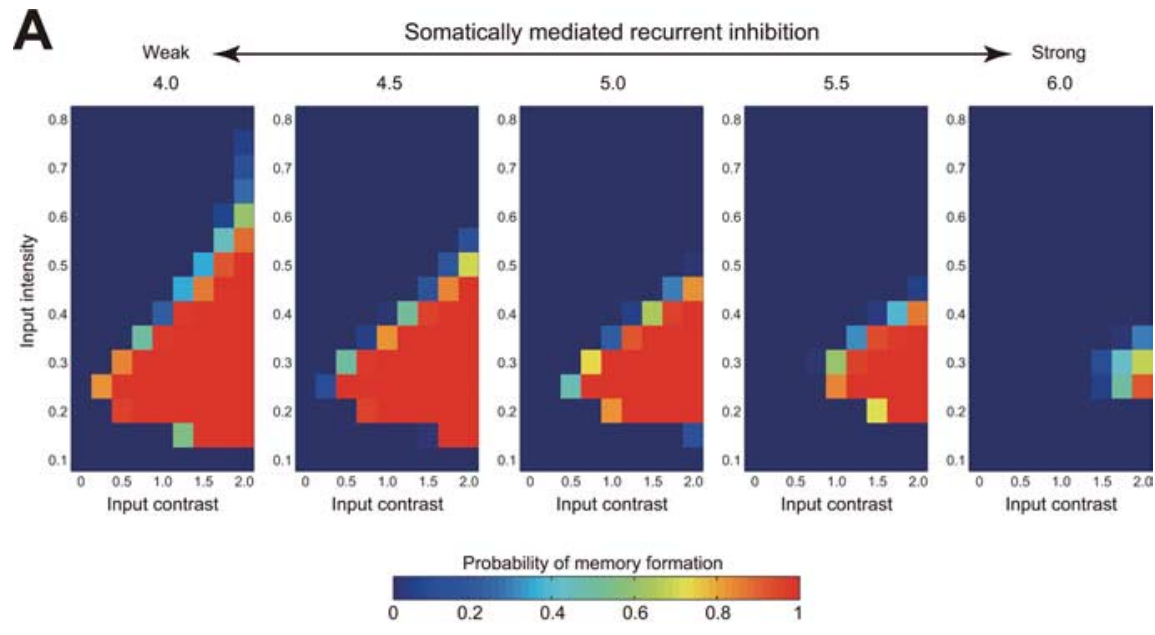

B
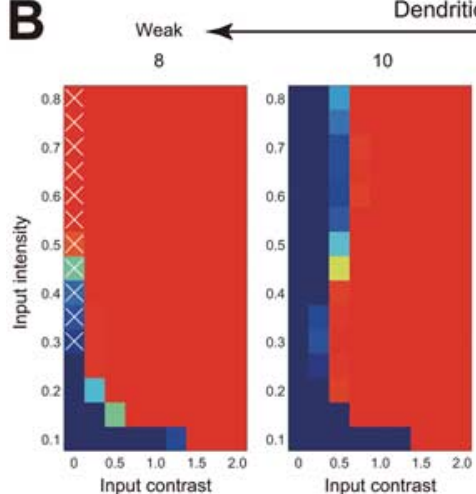

Dendritically mediated recurrent inhibition


Figure 8. Dependence of the circuit behavior on the parameters in the case of highly nonlinear dendrites. Dependence of the probability of memory formation (refer to the color bar) on the input contrast (horizontal axes) as well as on the input intensity (vertical axes) was examined for five different inhibition strengths. $\boldsymbol{A}$ and $\boldsymbol{B}$ show the cases of somatically mediated subtractive recurrent inhibition and the case of dendritically mediated divisive recurrent inhibition, respectively. The white crosses $(\boldsymbol{B}$, leftmost panel) indicate the formation of inaccurate memory [accuracy (Fig. $3 B, C,<0.98$ ]; otherwise the accuracy of memory was $>0.98$. As shown here, contrast-dependent accurate memory formation was observed for a range of input intensity with either type of recurrent inhibition. More precisely, the permitted range for input intensity was generally wider in the case of dendritically mediated recurrent inhibition $(\boldsymbol{B})$, especially when the inhibition strength was finely tuned (strength 10: second panel from the left). This seems reasonable because dendritically mediated recurrent inhibition, but not somatically mediated one, is considered to be able to compensate the change in the amount of feedforward excitation on each dendritic branch, although the compensation cannot be perfect because of the presence of the positive dendritic threshold.

Even if the number of compartments was further decreased to nine [Fig. $9 B c(i i i)$ ], the behavior of the circuit with dendritically mediated inhibition was mostly conserved (bottom row). However, in the case with somatically mediated inhibition (top row), contrast-dependent accurate memory formation was possible only for a limited range of input intensity $(\sim 0.2)$; memory was not formed or inaccurate memory could be induced for the input with different intensity. When the pyramidal cell had only three dendritic compartments [Fig. 9Bc(iv)], contrast-dependent accurate memory formation occurred only for a limited range of input intensity in both somatically or dendritically mediated inhibition cases. What caused such differences in the circuit behavior along with the decrease in the number of compartments? As I have shown above (Fig. 7), to prevent the formation of inaccurate memory in response to a low-contrast input, it seems essential that "most of" the dendritic branches do not have impact on somatic firing so that cell-to-cell difference in the amount of excitation onto those branches can be canceled. In the case in which the pyramidal cell had 27 compartments [Fig. 9Ba(ii)], the

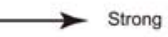

Strong

number of branches that received significant amount of feedforward excitation (visible in the figure) was 7 , and thus, such cancellation occurred on at least 20 of 27 (possibly 22 of 27 ) branches, that is, $>74 \%$ (possibly $81 \%$ ) of the branches. In contrast, if the pyramidal cell had only three compartments [Fig. 9Ba(iv)], the cancellation could occur on only two of three branches, or $67 \%$ of the branches, which is thereby supposed to be insufficient to prevent the undesirable competition. Note that, however, those percentage values themselves do not have direct meanings because our model is very simple, in particular, branches were all identical.

What happens if the branches are not identical? I examined the effect of the intercompartment heterogeneity in the model [the number of the compartments (branches) was again set to the original value (100) in the following]. Figure $10 B$ shows the circuit behaviors when the threshold of the moderately nonlinear dendrite, which was originally set to 0 (Fig. $1 \mathrm{Hb}$ ), was subject to random positive deviation (Fig. $10 A a$, green and blue curves). Subtractive dendritic recurrent inhibition was assumed as same as in the original simulations (Fig. 4). When the threshold was exactly at 0 (i.e., the circuit had the original moderately nonlinear dendrite), the circuit behavior was completely invariant for arbitrary input intensity weaker than a certain level (Fig. 10Ba) [above which the behavior varied because of the effect of the dendritic saturation, as expected above (Fig. 5)]. When the random positive deviation of the threshold was incorporated, such a genuine intensity semiinvariance could no longer hold (Fig. $10 B b, B c)$, although contrast-dependent memory formation was shown to still occur for a certain range of intensity. It turned out, therefore, that in order for the ideal contrastdependent intensity-invariant property to be realized, the dendritic threshold should be rather strictly tuned to 0 . It has been shown that summation of excitatory inputs applied onto the dendrite could become nearly linear possibly except for rectification attributable to branch-specific inhibition (Cash and Yuste, 1999), in particular, in the case with spatially dispersed and temporally asynchronous inputs (Gasparini and Magee, 2006), presumably by virtue of appropriately tuned dendritic active conductance (Cash and Yuste, 1999; Gasparini and Magee, 2006). Our model, however, describes the relationship between the inputs onto the dendritic branches and the output firing rate of the cell in a rather abstract manner, and therefore, whether such active linearization mechanisms could realize the required tuning of the dendritic threshold in our model to 0 would need to be studied by using spiking neuron models. Figure $10 \mathrm{C}$ shows the behavior of the circuit with the highly nonlinear dendrite including intercompartment heterogeneity, that is, when the threshold, slope, and upper bound (saturation) of the dendritic nonlinearity were all 


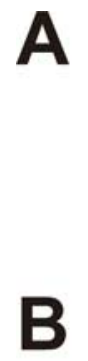

a Model

(i) 135 compartments

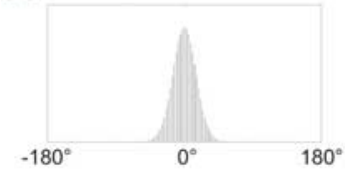

(ii) 27 compartments

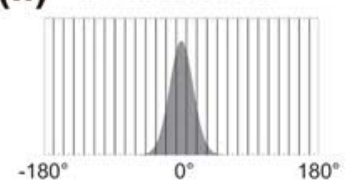

(iii)

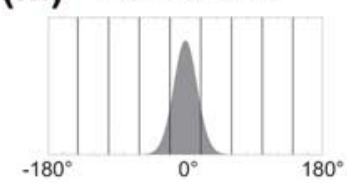

b Moderately nonlinear dendrite
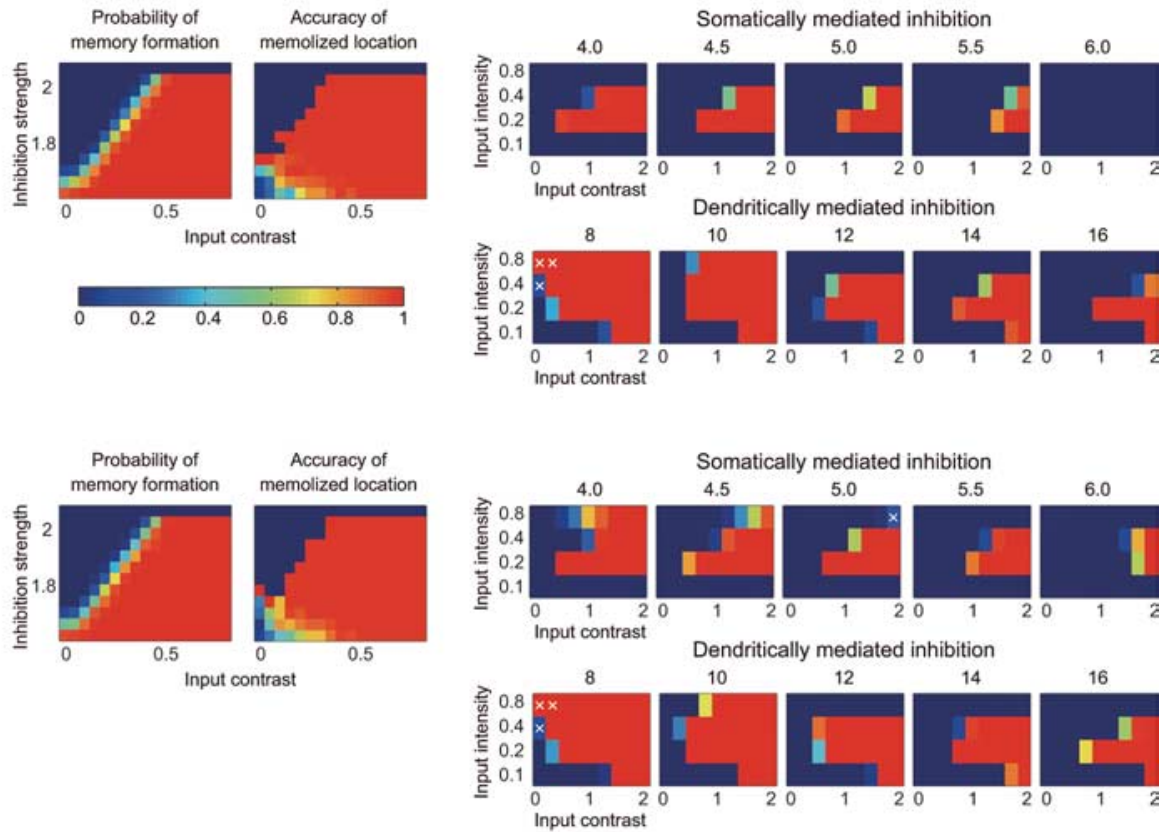

C

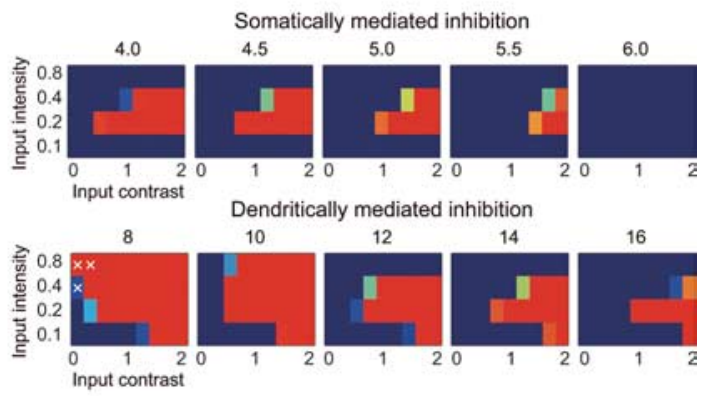

Highly nonlinear dendrite b

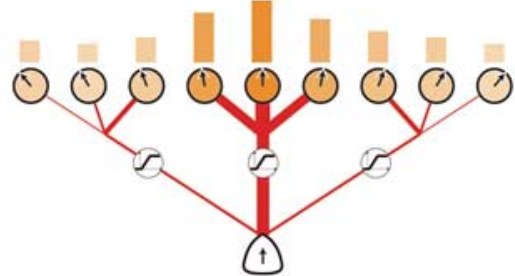


subject to random variations as shown in Figure 10, $A b$ and $A c$. Either somatically (Fig. 10 Ca) or dendritically (Fig. 10Cb) mediated global recurrent inhibition was considered. In both cases, as the variation in the dendritic nonlinearity increased (from the top rows to the bottom rows in Fig. $10 \mathrm{Ca}, \mathrm{Cb}$ ), memory became easier to be formed, and the tuning curves of individual neurons became heterogeneous as shown in Figure 10, $C c$ and $C d$. Meanwhile, the accuracy of the memory was decreased, as indicated by the black and white crosses in Figure 10, $C a$ and $C b[<0.95$ (but $>0.9$ ) and $<0.9$ accuracy, respectively]. However, the decrease in the accuracy appeared to be gradual, rather than abrupt, as shown in Figure 10, Ce and Cf. Therefore, the overall feature of the circuit with the highly nonlinear dendrite discussed above can be said to be fairly robust against such intercompartment heterogeneity.

\section{On the nature of recurrent inhibition}

Regarding the source of recurrent inhibition during working memory-associated stimulus-selective sustained activity, it was suggested that the fast-spiking (FS) cell could be a candidate (Rao et al., 1999, 2000; Constantinidis et al., 2002). However, based on the findings that synapses arriving at or coming from FS cells show strong short-term depression whereas synapses for certain types of non-FS cells rather show short-term facilitation (Reyes et al., 1998; Gibson et al., 1999; Beierlein et al., 2003; Gao et al., 2003; González-Burgos et al., 2004, 2005), it was also proposed that those non-FS interneurons could be an ideal candidate for the source of recurrent inhibition in the working memory circuit (González-Burgos et al., 2005). However, the possibility for the FS cells has not be denied (Galarreta and Hestrin, 1998; Goldman et al., 2002), and thus the exact cell type(s) that mediate recurrent inhibition in the spatial working memory circuit seems to remain elusive. Because there is a huge variety of GABAergic interneurons, some of which target the soma of pyramidal cells and most of which target various domains of the pyramidal dendrites with various degree of preference (Kawaguchi and Kubota, 1997; Somogyi et al., 1998; Markram et al., 2004), there seem to be many possibilities (see below for more specific arguments). One point regarding the nature of recurrent inhibition that was not considered well in the above simulations is the effective range. I have so far assumed that both somatically and dendritically mediated recurrent inhibition operate globally (i.e., the same impact to all the pyramidal cells). Whereas certain types of soma-targeting

\section{$\leftarrow$}

(Figure legend continued.) input layer neuron, of a given pyramidal cell was assumed to have a bell-shaped distribution, in the same manner as before (Fig. 1G). In (ii)-(iv), each thin rectangular indicates each single compartment, and the area of the bell-shaped distribution included in each rectangular indicates the weight of the feedforward excitation on the corresponding compartment. Note that only three compartments received significant amount of feedforward excitatory inputs (one compartment received most of them and the neighboring two received the rest) in the case of total nine compartments [(iii)] and only a single compartment received feedforward excitation in the case of total three compartments [(iv)]. $\boldsymbol{B} \boldsymbol{b}$, Behaviors of the circuit with moderately nonlinear dendrite and dendritically mediated recurrent inhibition. The configurations are the same as in Figure 4D. Overall tendency did not strongly depend on the number of compartments, indicating that the main feature of the circuit holds even when the pyramidal cell has only a few parallel dendritic compartments for independent nonlinear input integration. $B \boldsymbol{C}$, Behaviors of the circuit with highly nonlinear dendrite and either somatically (top rows) or dendritically (bottom rows) mediated recurrent inhibition. The configurations are the same as in Figure 8, except that the vertical axes are in the log scale. Circuit behavior depended on the number of compartments more strongly than the case of moderately nonlinear dendrite $(\boldsymbol{B} \boldsymbol{b})$. However, even when there were only nine compartments [(iii)], contrast-dependent accurate memory formation was still observed for a range of input intensity in the case with dendritically mediated recurrent inhibition (bottom row). cells such as large basket cells have horizontally and densely extended axonal arborization that is considered to be able to mediate recurrent inhibition over the wide range, other interneurons, especially, those targeting dendrites would have more vertically oriented axons so that their effects would be more local. Therefore, next I examined how the circuit behaves if dendritic recurrent inhibition operates more locally. Figure $11 \mathrm{Ba}$ shows the circuit behavior when dendritic recurrent inhibition operates locally with the range three times wider than that of the feedforward and recurrent excitation, as shown in the blue line (a) in Figure $11 \mathrm{~A}$. With such moderately local dendritic recurrent inhibition, although multiple bumps (Fig. 11C) were shown to be easily formed under certain conditions (indicated by white diamonds in Fig. 11 B), the circuit feature that I have so far discussed, namely, contrast-dependent intensity-insensitive accurate memory formation was shown to still occur when the inhibition strength was appropriately tuned (26). It would be reasonable; if the circuit is in a state in which every pyramidal cell has comparable level of small activity, dendritic recurrent inhibition that each cell receives is expected to become comparable regardless of whether it is driven by all the pyramidal cells or instead by some portion of them, unless their number is too small. When the inhibition range became narrower, namely, twice wider than the excitation (Fig. 11 $A b$ ), however, multiple bumps were more easily formed and the contrast-dependent intensity-insensitive accurate memory formation could no longer occur (Fig. $11 \mathrm{Bb}$ ).

In all the simulations I have thus far shown, either somatically or dendritically mediated recurrent inhibition was considered only in an isolated manner. In the actual circuit in the brain, however, it would be more likely that those two types of recurrent inhibitions exist together. Therefore, here let us see how the circuit behaves with such a combination of somatic and dendritic recurrent inhibition. When globally operated somatic recurrent inhibition and also globally operated dendritic recurrent inhibition coexist in the circuit with the highly nonlinear dendrite (Fig. $12 \mathrm{~A}$ ), the circuit behavior appeared to interpolate those in the extremes cases in which only one of those two types of inhibition exists (Fig. $8 A, B$ ). It was therefore confirmed that the phenomena I have shown in the above are not specific to biologically unrealistic situations in which exclusively somatic or dendritic recurrent inhibition exists. It would be more intriguing to see what happens when globally operated somatic recurrent inhibition, presumably mediated by certain types of FS cells, is coupled with locally operated dendritic one. Figure $12 \mathrm{~B}$ shows the circuit behavior when local dendritic inhibition whose width is three times wider than the excitation (Fig. 11Aa) (same as considered in Fig. $11 \mathrm{Ba}$ ) was coupled with global somatic inhibition. As shown in Figure 12B, contrast-dependent intensity-insensitive accurate memory formation occurred under certain conditions. Notably, the permitted range of the dendritic inhibition strength for such a desirable response appeared wider than the case with local dendritic inhibition only (Fig. $11 B a$ ). It seems that there are functional distinctions; whereas globally operated somatic recurrent inhibition ensures the uniqueness of the bump (i.e., avoids the appearance of multiple bumps), locally operated dendritic recurrent inhibition mainly serves for preventing the formation of inappropriate bump (inaccurate memory) in the case of lowcontrast input. Even narrower dendritic recurrent inhibition (Fig. 11Ac,Ad), which by itself could not realize contrastdependent intensity-insensitive accurate memory formation (Fig. $11 \mathrm{Bb}$ ), was shown to realize such a response when it was coupled with global somatic recurrent inhibition (Fig. 12C,D). Note that, when the inhibition strength (especially that of den- 
dritic inhibition) was weak, although contrast-dependent intensity-insensitive accurate memory formation was observed, the sustained activity bump appeared to be too wide (black circles in the figures indicate the case in which the bump included more than the one-half of the pyramidal cells).

Figure $13 A$ shows the circuit behavior in the same condition as Figure $12 C$ except that the variation in the dendritic nonlinearity such as shown in Figure $10 A b$ was incorporated. In consequence, the memory accuracy was decreased, but not drastically; the accuracy was shown to be $>0.9$ in most conditions [the black crosses indicate $>0.9$ (but $<0.95$ ) accuracy in the figure]. Figure $13 B$ shows the circuit behavior also in the same condition as Figure $12 C$ except that the local dendritic recurrent inhibition was assumed to be recruited only above a certain threshold (for details, see Materials and Methods), which may exist but has not so far been incorporated in our model. In the results, memory became slightly easier to be formed as expected, but the capability of contrastdependent intensity-insensitive accurate memory formation was preserved. It was also mostly preserved when both the variation in the dendritic nonlinearity and the threshold for dendritic recurrent inhibition were incorporated (Fig. 13C). It should be noted, however, that the threshold for inhibition assumed in those simulations (Fig. 13 B, C) was not very high so that recurrent inhibition was recruited not only when the localized high-level activity bump was formed but also when the most pyramidal cells had comparable relatively low or moderate-level activities, and importantly, that such a recruitment in the latter case appeared to be essential for preventing inappropriate memory formation in response to high-intensity low-contrast input and thereby realizing intensityinsensitive accurate memory formation (Figs. 5, 7). For this reason, it is unlikely that the dendritic recurrent inhibition considered in the present study represents Martinotti or somatostatin-expressing cell-mediated inhibition that was recently found in the somatosensory cortex (Kapfer et al., 2007; Silberberg and Markram, 2007), because Martinotti cell was shown to be recruited only after it receives high-frequency input from single pyramidal cell via prominently facilitating synapses. Instead, dendritic recurrent inhibition assumed in the present study can be implemented by many other cell types, considering that there is a huge variety of neocortical GABAergic interneurons
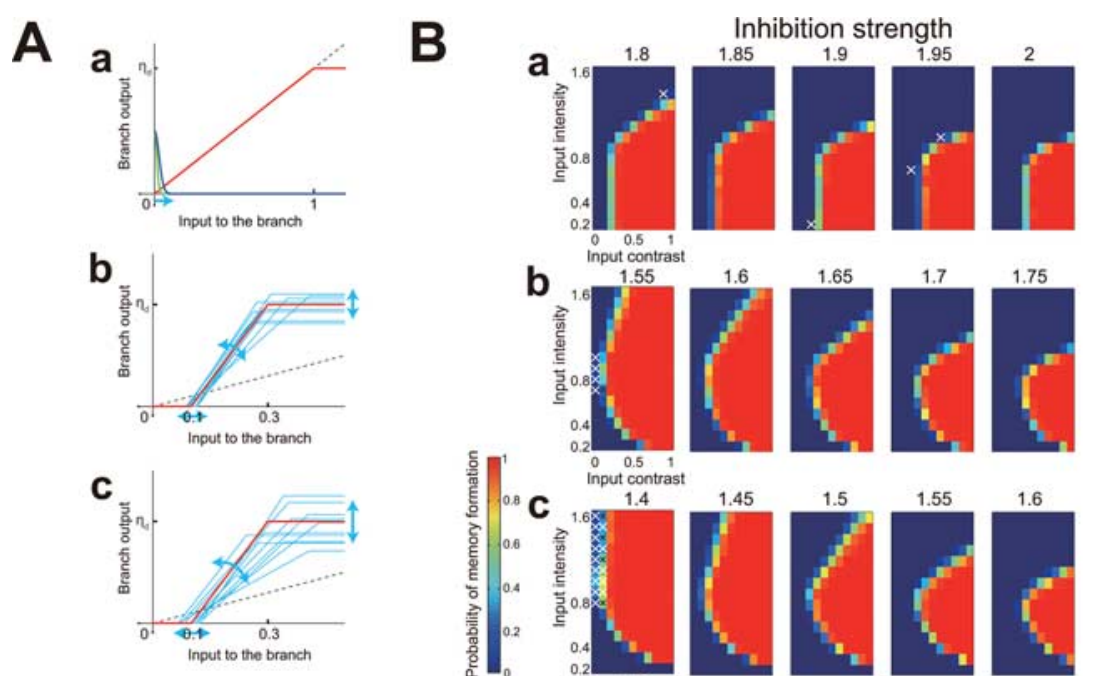

b


Somatic Inhibition
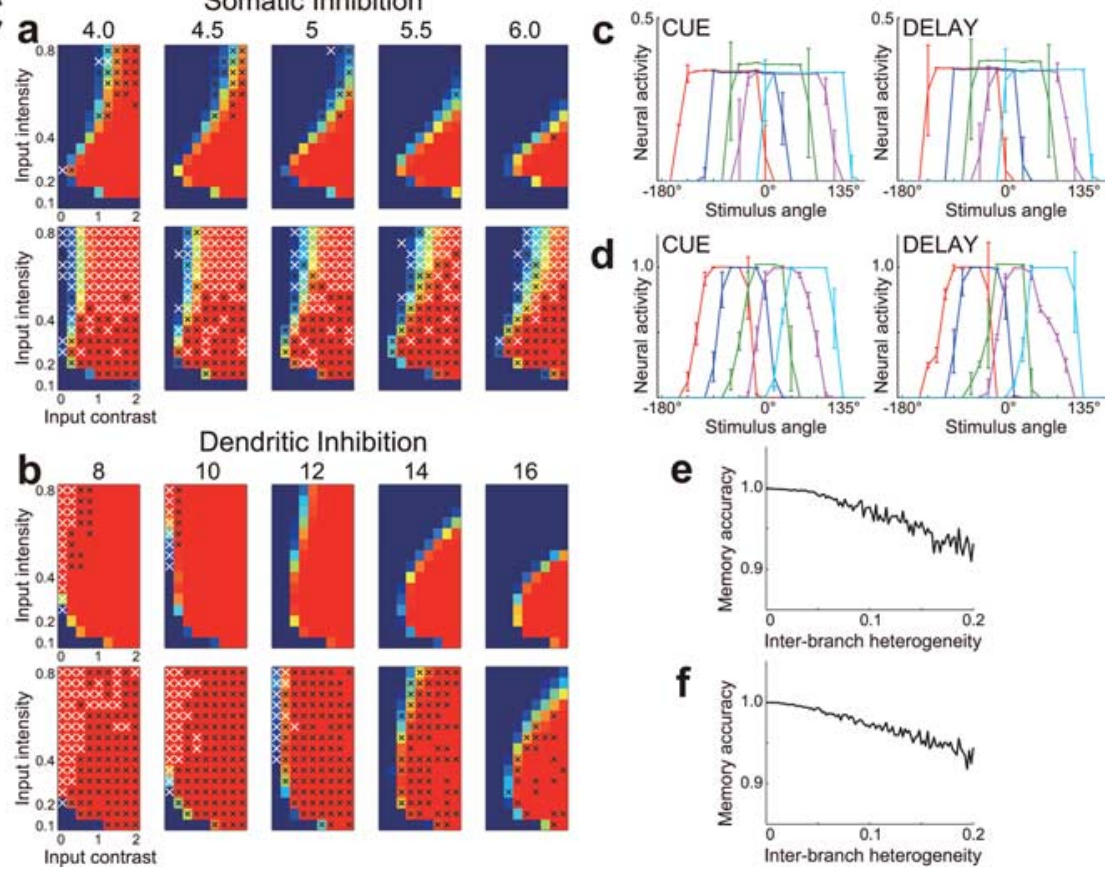

Figure 10. Effects of the intercompartment heterogeneity. Aa, The threshold of the moderately nonlinear dendrite was subject to two levels of random positive deviations. The red line shows the input integration of the original moderately nonlinear dendrite (the same as in Fig. $1 \mathrm{Hb}$ ) and the left bottom green and blue curves indicate the presumed distributions of the dendritic threshold; positive parts of Gaussians with the standard variation of 0.02 and 0.04 , respectively. $\boldsymbol{A} \boldsymbol{b}, \boldsymbol{A c}$, Random variations in the threshold, slope, and upper bound (saturation) were incorporated into the highly nonlinear dendrite. The red line shows the input integration of the original highly nonlinear dendrite (the same as in Fig. $1 \mathrm{Hc}$, but in a different aspect ratio) and the light blue lines indicate 10 example curves incorporating the variations whose SDs are one-tenth $(\boldsymbol{A} \boldsymbol{b})$ or one-fifth $(\boldsymbol{A c})$ of the means, respectively. $B a-B C$, Circuit behavior of the original moderately nonlinear dendrite case $(B a)$ and the cases with two levels of positive deviations of the dendritic threshold ( $\boldsymbol{A} \boldsymbol{a}$, green and blue curves, $\boldsymbol{B} \boldsymbol{b}, \boldsymbol{B} \boldsymbol{c})$. Subtractive dendritic recurrent inhibition was assumed, the same as in Figure 4. The configurations are the same as in Figure 8, except that the white crosses indicate $<0.9$ accuracy (rather than $<0.98$ accuracy as in the previous figures). Genuine intensity invariance below a certain level held in the original moderately nonlinear dendrite case $(\mathbf{B a})$ but not in the cases with positive deviations of the threshold $(\boldsymbol{B} \boldsymbol{b}, \boldsymbol{B} \boldsymbol{C})$, although contrast-dependent memory formation was shown to still occur for a certain range of intensity. $C a, C b$, Behavior of the circuit with the highly nonlinear dendrite incorporating two levels of variations ( $\boldsymbol{A} \boldsymbol{b}$, top rows; $\boldsymbol{A} \boldsymbol{c}$, bottom rows). Somatically $(\boldsymbol{C} \boldsymbol{a})$ or dendritically $(\boldsymbol{C} \boldsymbol{b})$ mediated recurrent inhibition was considered. The configurations are the same as in $\boldsymbol{B}$; in addition, the black crosses indicate $<0.95$ (but $>0.9$ ) accuracy. As the variation increased, memory became easier to be formed, while the memory accuracy was decreased as indicated by the crosses. $\boldsymbol{C}, \boldsymbol{C d}$, Examples of the tuning curve of individual neurons in the circuit with somatic (Cc) or dendritic (Cd) recurrent inhibition in the case with the interbranch heterogeneity 0.1 . The error bars indicate the mean and the SD over 100 input (stimulus) at each of the 24 angles (horizontal axis: $-180,-165, \ldots, 165^{\circ}$ ). The input intensity was 0.25 and the contrast was 0.75. Tuning curves of five neurons in the presence of (left: time 200) or after the disappearance of (right: time 400) the cue stimulus are shown. $(\boldsymbol{e}, \mathbf{C}$, Dependence of the memory accuracy on the level of the interbranch heterogeneity in the somatic $(\boldsymbol{C e})$ or the dendritic ( $\boldsymbol{C}$ ) recurrent inhibition cases at the fixed intensity $(0.25)$ and the contrast (0.75). The decrease in the accuracy appeared to be gradual, rather than abrupt. 

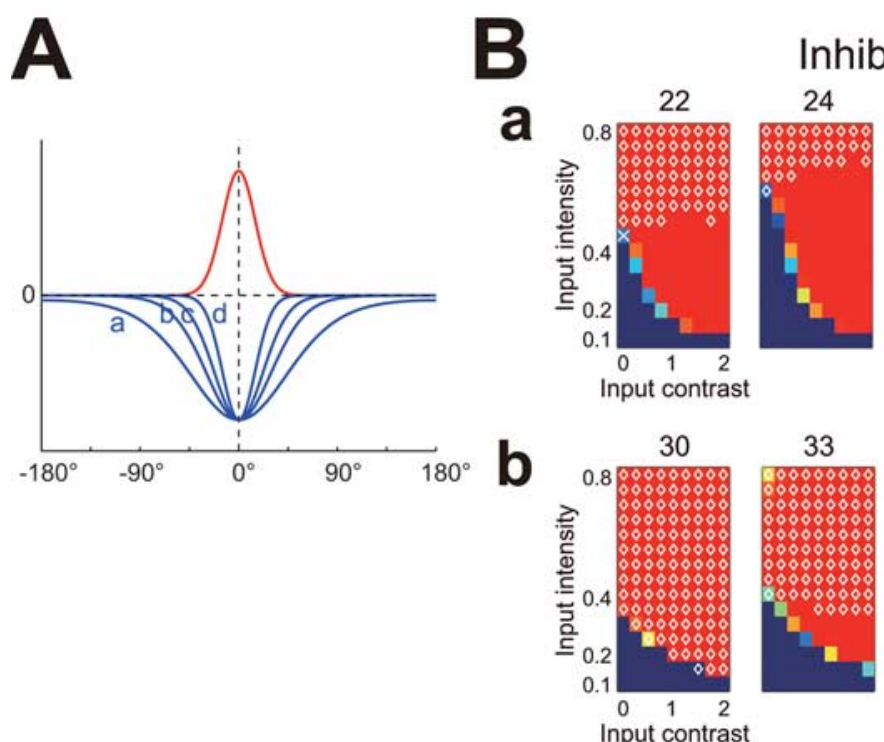

Inhibition strength
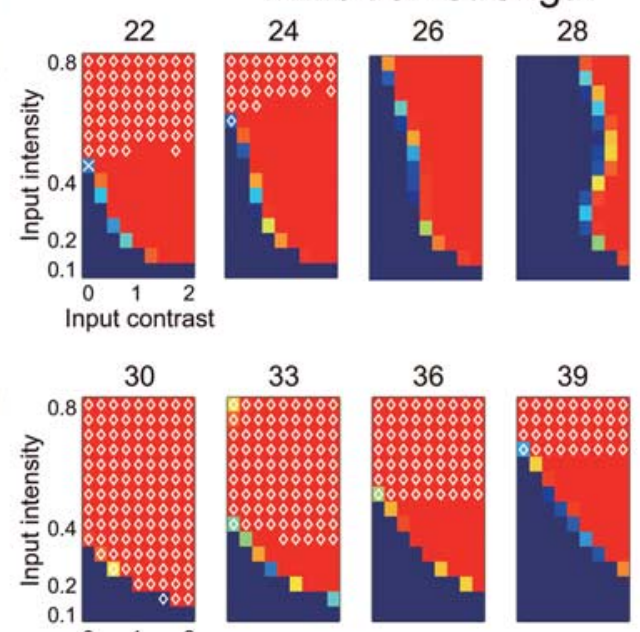

30

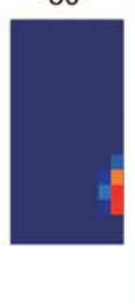

42

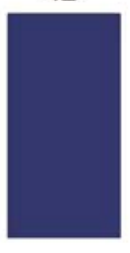

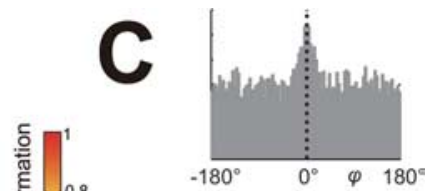
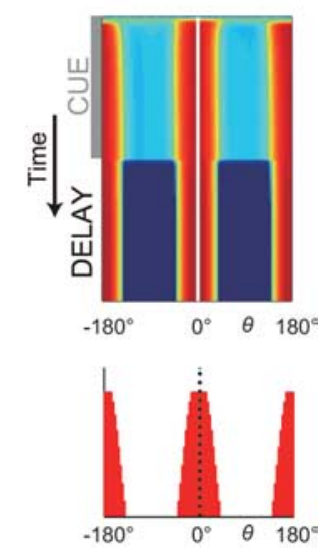

Figure 11. Behavior of the circuit with local dendritic recurrent inhibition. $A$, The ranges of the excitation and inhibition. The red line indicates the presumed range of the feedforward and the recurrent excitation. The four blue lines indicate the presumed ranges of the dendritically mediated local recurrent inhibition: three, two, or 1.5 times wider than (a-c), or the same range as (d), the excitation, respectively. $\boldsymbol{B} \boldsymbol{a}, \boldsymbol{B} \boldsymbol{b}$, Circuit behavior when the local dendritic recurrent inhibition was three $(\boldsymbol{B} \boldsymbol{a})$ or two $(\boldsymbol{B} \boldsymbol{b})$ times wider than the excitation (corresponding to the blue lines a and b in A), respectively. The configurations are the same as in Figure $10 C$ : the black and white crosses indicate $<0.95$ (but $>0.9$ ) and $<0.9$ accuracy, respectively; in addition, the white diamonds indicate the conditions in which the formation of multiple bumps was observed. When the dendritic inhibition was not very narrow $(\mathbf{B a})$, contrast-dependent intensity-insensitive accurate memory formation occurred at an appropriate inhibition strength (26). When the inhibition was made further narrower (Bb), however, multiple bumps were more easily formed and the contrast-dependent intensity-insensitive accurate memory formation could no longer occur. C, An example trial in which two activity bumps were formed and sustained.

(Kawaguchi and Kubota, 1997; Somogyi et al., 1998; Markram et al., 2004) and most of them target pyramidal dendrites with various degrees of preference [Kubota et al. (2007), their Fig. 4] and also many of them have depressing synapses (Gupta et al., 2000), contrary to Martinotti cells. Among them, I would like to suggest that basket cells can also be a candidate, given that many of them receive excitation from pyramidal cells via depressing synapses, and moreover, despite their being known as as soma-targeting cells, many basket cells also prevalently target basal/proximal dendrites of pyramidal cells, including medial or even distal branches [Wang et al. (2002), their Fig. 5; or Gupta et al. (2000), their Fig. 6C; but see Somogyi et al. (1998), their Fig. 7B; whether or not to target medial/distal branches would depend on the subtype, brain region, etc.]. Considering that inhibitory input applied onto a basal dendritic branch was shown to have a branch-specific effect by a detailed simulation study (Rhodes, 2006), together with an indication derived from a direct experiment in the pyramidal basal dendrites (Nevian et al., 2007) that the basal dendrites should be considered as a separate integrative compartment, rather than being regarded as a single basalsomatic region, despite their proximity to the soma (although inhibition was not examined in that study), it seems likely that certain portion of the synapses made by basket cells onto the pyramidal basal dendrites can have branch-specific effect, and therefore, that basket cells can implement not only somatic inhibition but also dendritic inhibition assumed in the present study at least for the basal dendrite. Notably, it is possible that the basal dendrite is the main target of recurrent excitatory inputs as discussed before, and branch-specific inhibition of recurrent excitation is crucial for the intensity-insensitive contrast-dependent accurate memory formation in the model of the present study (Figs. 5, 7). Speaking more specifically about the cell type, large basket cells could implement global somatic and dendritic recurrent inhibition, whereas nest and/or small basket cells could implement local recurrent inhibition. Regarding the apical den- drites, although basket cells do make synapses on the apical, oblique, and even tuft dendrites [Wang et al. (2002), their Fig. 5], other types of interneurons, such as (subtypes of) bitufted cells (Markram et al., 2004) or calbindin-positive interneurons with ascending axons observed in the monkey prefrontal cortex (Zaitsev et al., 2005), for example, would implement branch-specific inhibition on the distal apical/tuft dendrites (for more detailed description on this matter, see Materials and Methods, Network architecture). Figure $13 D$ shows the circuit behavior when recurrent excitation was assumed to be nonuniformly distributed over the branches (Fig. 1G, red dashed line) (for details, see Materials and Methods), exploring the possibility that the dendritic plasticity rule similar to what was assumed for the feedforward excitation is also applied to the recurrent excitation, although to less extent (as discussed before). As shown in Figure 13D, the capability of contrast-dependent intensity-insensitive accurate memory formation was preserved. It was also preserved when the strength of recurrent excitation was increased (Fig. 13E) or decreased (Fig. 13F), with the permitted ranges of inhibition strengths changed accordingly. More precisely, increasing recurrent excitation, which means decreasing the dendritic threshold relatively, resulted in better performance of the circuit in terms of contrast-dependent intensity-insensitive accurate memory formation (Fig. 13E), whereas decreasing recurrent excitation appeared to have the opposite effect (Fig. 13F).

As shown in the above, when local dendritic recurrent inhibition was combined with global somatic recurrent inhibition, contrast-dependent intensity-insensitive accurate memory formation was realized as easily as the case with global dendritic recurrent inhibition (Fig. 8B). It is possibly because such local dendritic inhibition can effectively balance out local activity peaks, unless the peak is really significant, so as to prevent inappropriate bump formation (inaccurate memory). Considering that global dendritic recurrent inhibition may cost much in terms of energy consumption and wiring, combining local dendritic 

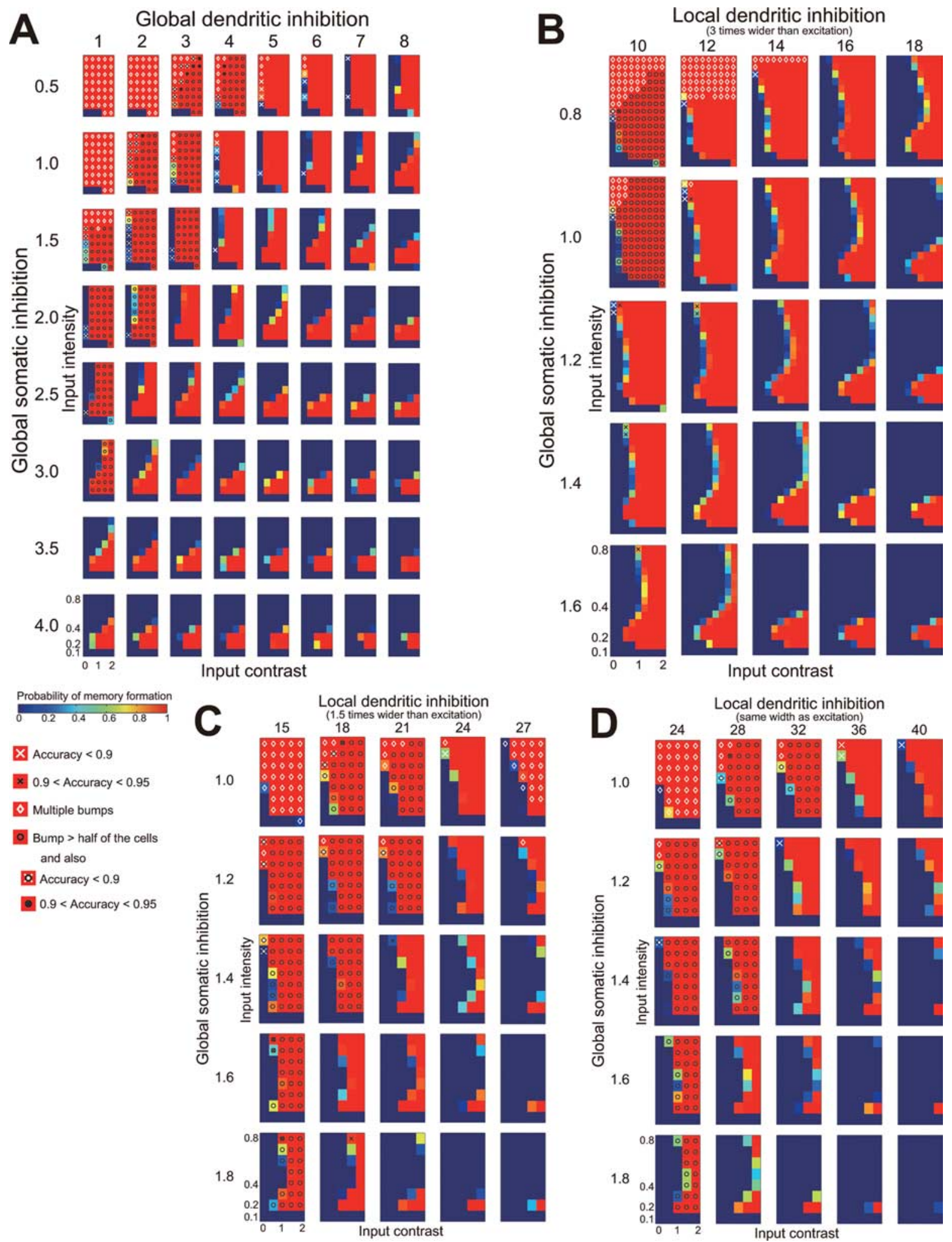

Figure 12. Behavior of the circuit with both somatically and dendritically mediated recurrent inhibition. $A-D$, Circuit behavior under several conditions with both somatically and dendritically mediated recurrent inhibition. The configurations are the same as in Figure $11 B$ : the black and white crosses indicate $<0.95$ (but $>0.9$ ) and $<0.9$ accuracy, respectively, and the white diamonds indicate the conditions in which the formation of multiple bumps was observed; in addition, the black circles indicate the cases in which the sustained activity bump includes more than the one-half of the pyramidal cells (it would be too wide). $A$, Circuit behavior with globally operated somatic and dendritic recurrent inhibitions. The behavior appeared to interpolate the extremes cases in which only one of those two types of inhibition existed (Fig. $8 A, B) . \boldsymbol{B}-\boldsymbol{D}$, Circuit behavior when globally operated somatic recurrent inhibition was coupled with locally operated dendritic one, whose range was three $(\boldsymbol{B})$ or $1.5(\boldsymbol{C})$ times wider than, or the same as $(\boldsymbol{D})$, that of the feedforward and recurrent excitation (corresponding to Fig. $11 A a, A c, A d$, respectively). Contrast-dependent intensityinsensitive accurate memory formation occurred under certain conditions; the permitted range of the dendritic inhibition strength appeared to be wider than the cases with local dendritic inhibition only (compare $\boldsymbol{B}$ with Fig. 11 Ba). Fairly narrow dendritic recurrent inhibition $(\boldsymbol{C}, \boldsymbol{D})$, which could not achieve contrast-dependent intensity-insensitive accurate memory formation by itself, became able to achieve such a response by virtue of the coexisting global somatic inhibition. 
A

1.0

With inter-branch heterogeneity

Local dendritic inhibition

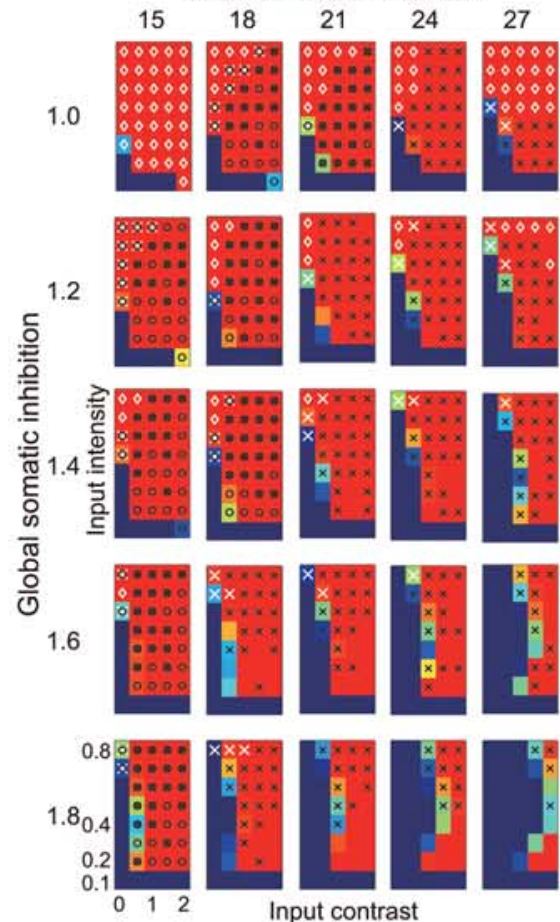

Probability of memory formation

\begin{tabular}{|lllll}
\hline 0 & 0.2 & 0.4 & 0.6 & 0.8
\end{tabular}
B.

With threshold for dendritic inhibition Local dendritic inhibition

1.0


Input contrast
With inter-branch heterogeneity \&
threshold for dendritic inhibition

Local dendritic inhibition

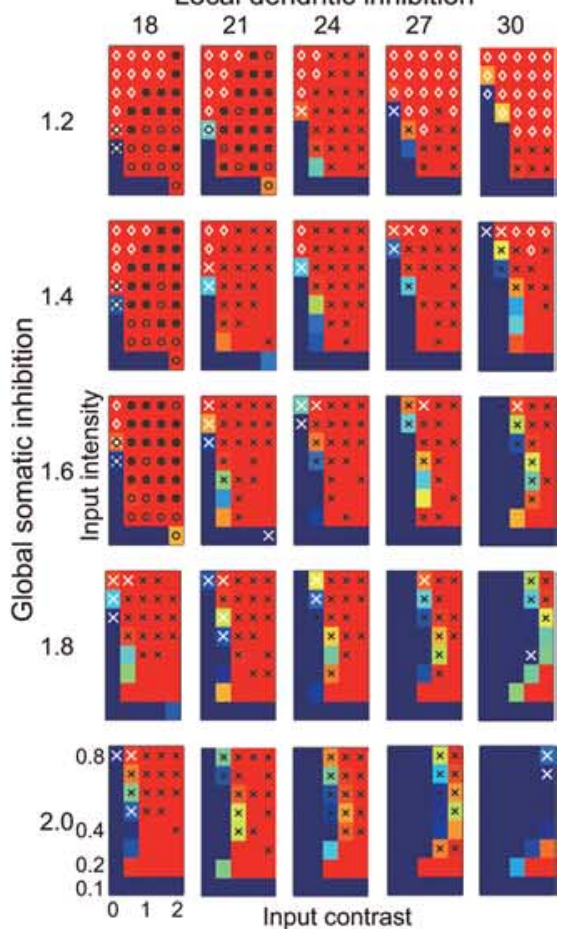

[C.] Accuracy $<0.9$

$0.9<$ Accuracy $<0.95$
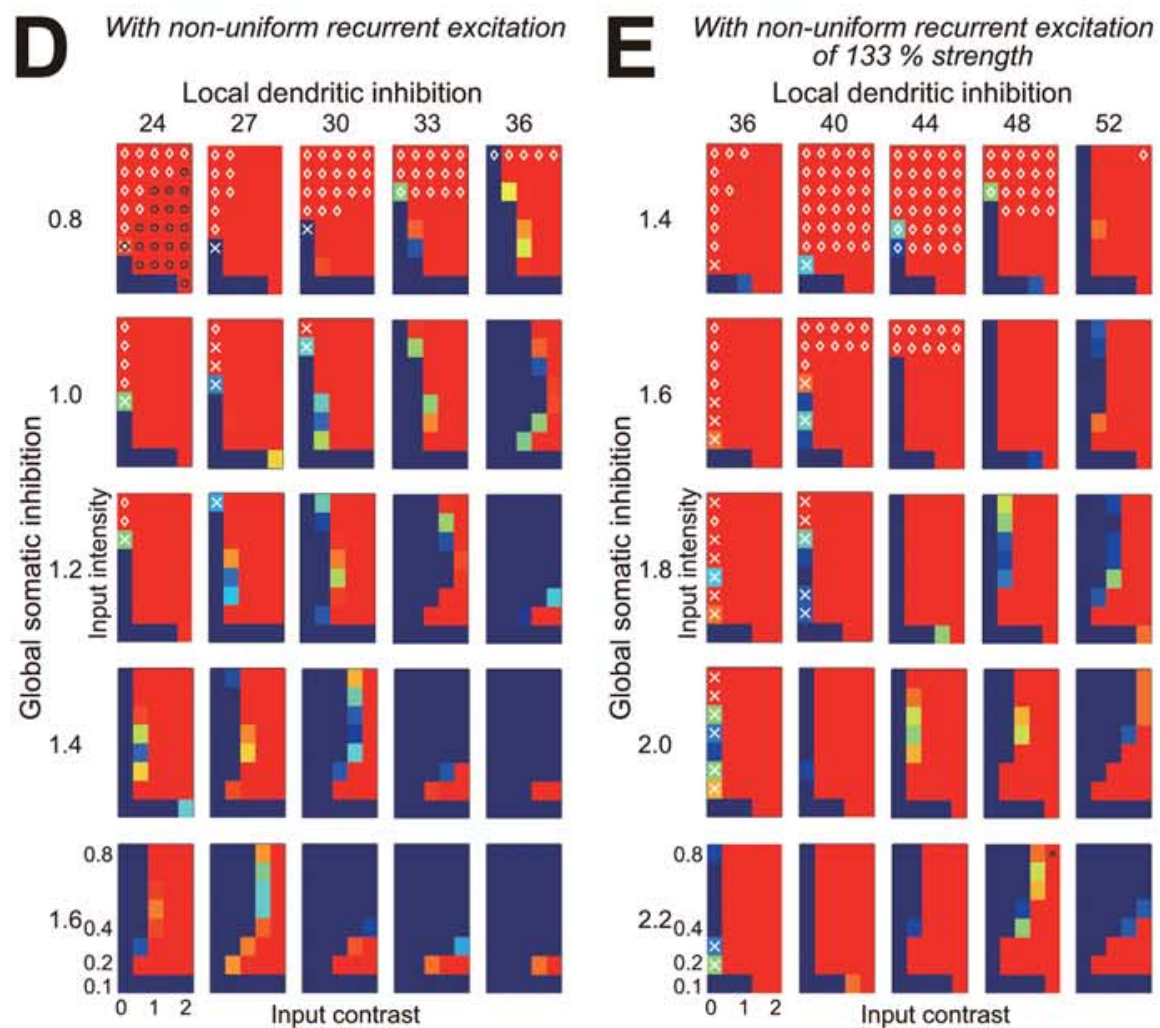

W. With non-uniform recurrent excitation Local dendritic inhibition
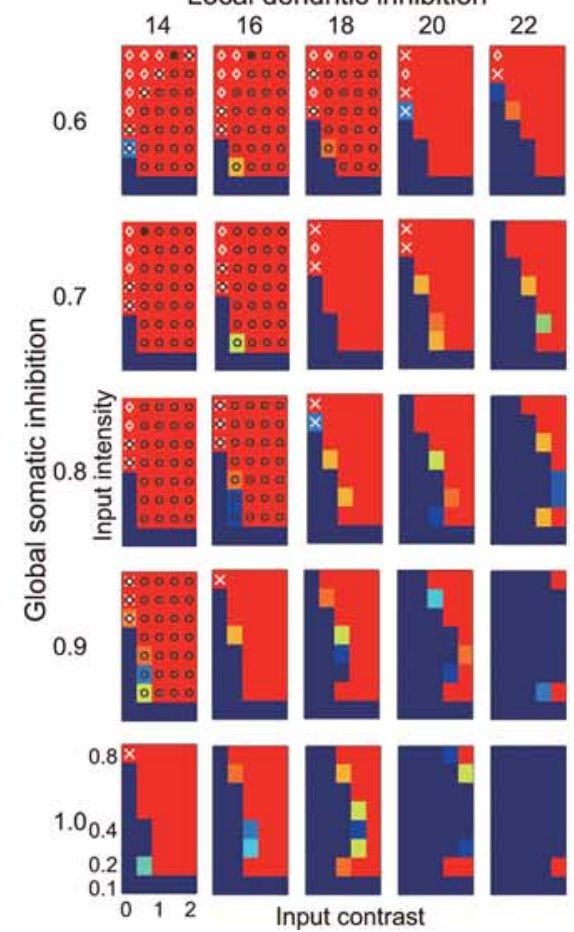

Figure 13. Main feature of the circuit behavior survived after moderate modifications of the model. $\boldsymbol{A}-\boldsymbol{D}$, Circuit behavior with global somatic recurrent inhibition and local dendritic recurrent inhibition whose range was 1.5 times wider than the excitation (Fig. 11 Ac); the same condition as Figure 12 Cexcept that some modifications have been made onto the model. The configurations are the same as in Figure 12; the black and white crosses indicate $<0.95$ (but $>0.9$ ) and $<0.9$ accuracy, respectively; the white diamonds indicate the conditions in which the formation of multiple bumps was observed; the black circles indicate the cases in which the sustained activity bump includes more than the one-half of the pyramidal cells (too wide). $\boldsymbol{A}$, Variations in the dendritic nonlinearity such as shown in Figure $10 \mathrm{Ab}$ was incorporated. Contrast-dependent intensity-insensitive memory formation with $>0.9$ accuracy still occurred for ranges of recurrent inhibition strengths. $\boldsymbol{B}$, The local dendritic recurrent inhibition was assumed to be recruited only above a certain threshold. Memory became easier to be formed, but (Figure legend continues.) 
inhibition with global somatic inhibition would be a better strategy. In the case of high-contrast input, however, local inhibition seems problematic for the formation and/or the maintenance of a correct memory. It could be ideally overwhelmed by local disinhibition (Wang et al., 2004; Carter and Wang, 2007), especially if the threshold for disinhibition is higher than that for inhibition (i.e., if local disinhibition is recruited only when a bump is formed, possibly via facilitating synaptic transmissions, whereas local inhibition is recruited even when, or possibly only when, a bump is not formed, via strong, reliable, but depressing synapses such as those observed between pyramidal cells and certain basket cells as well as other interneurons) (Gupta et al., 2000). However, Martinotti cell-mediated recurrent inhibition, which was recently found in the somatosensory cortex (Kapfer et al., 2007; Silberberg and Markram, 2007) and might also exist in the prefrontal/parietal spatial working memory circuits, seems to differ from such a "low-threshold" dendritic recurrent inhibition, because of the facilitating nature of the pyramidal-to-Martinotti synapses as mentioned above. Instead, considering that most Martinotti cells dominantly target apical, rather than basal, dendrites of pyramidal cells and that there might be an input domain specification such that external inputs mainly target apical/distal dendrites whereas recurrent inputs target basal/proximal dendrites as discussed before (Fig. 1G), Martinotti cell-mediated recurrent inhibition might specifically block the external input that comes after an activity bump (a memory) is formed, so as to increase the robustness of the memory against forthcoming distracting stimuli, as suggested by a previous study (Wang et al., 2004) with even more specificity to the external inputs given the spatial separation of the apical and basal dendritic compartments. Another possibility, emerging from the recent finding that Martinotti cells frequently make synapses on the pyramidal dendritic spines that receive thalamocortical, rather than intracortical, inputs (Kubota et al., 2007), is that they regulate inputs from the thalamus and thus are involved in the control of the working memory circuit by the basal ganglia or other subcortical areas via thalamus. Those features are expected to be incorporated into a model in the future to fully elucidate the functional repertoire conferred by the huge variety of inhibitory interneurons.

\section{Discussion}

By modeling the spatial working memory circuit incorporating multiple dendritic branches of individual pyramidal cells, with the assumption that external feedforward inputs are applied onto some portion of branches while recurrent inputs are distributed over branches, I have shown that dendritic compartmentalization could endow the circuit with the ability of contrastdependent intensity-insensitive accurate memory formation. If the dendritic integration follows moderate nonlinearity with the threshold finely tuned at 0 and there exists globally operated dendritic recurrent inhibition, genuine intensity semiinvariance

\footnotetext{
$\leftarrow$

(Figure legend continued.) contrast-dependent intensity-insensitive accurate memory formation still occurred. $\boldsymbol{C}$, Both the variations in the dendritic nonlinearity and the threshold for dendritic recurrent inhibition were incorporated. Note that the ranges of the inhibition strengths are different from $\boldsymbol{A}$ and $\boldsymbol{B}$. D, Recurrent excitation was assumed to be nonuniformly distributed over the branches as shown in the red dashed line in Figure 1G. The capability of contrast-dependent intensity-insensitive accurate memory formation was preserved. $\boldsymbol{E}, \boldsymbol{F}$, The same as $\boldsymbol{D}$ except that the strength of recurrent excitation was $33 \%$ increased $(\boldsymbol{E})$ or $20 \%$ decreased $(\boldsymbol{F})$. Increasing recurrent excitation $(\boldsymbol{E})$, which means decreasing the dendritic threshold relatively, resulted in better performance of the circuit in terms of contrastdependent intensity-insensitive accurate memory formation.
}

was realized (Figs. 4, $10 \mathrm{Ba}$ ), although whether and how those tunings could be biologically implemented remains to be seen. Even out of such an ideal condition, however, I have shown that the circuit with dendritic compartmentalization could realize contrast-dependent accurate memory formation for at least a certain range of intensity under rather wide conditions, in particular when local dendritic recurrent inhibition is coupled with global somatic recurrent inhibition (Figs. 12, 13). [In the above simulations, external input was assumed not to change with time. I have also examined the case in which input has time-varying noisy component, confirming that the results mostly did not change (Fig. 14) (see Materials and Methods).]

Without dendritic compartmentalization, recurrent inhibition itself possesses the ability to implement input normalization, as proposed in the models of sensory processing circuits (Carandini and Heeger, 1994; Hahnloser et al., 2000). Different from sensory processing, however, working memory is undebatably an attractor-based strongly nonlinear process, and thus, such conventionally considered mechanisms seem unable to work without additional elaboration, for which I propose dendritic compartmentalization here. Richer branches or spines of the pyramidal cellular dendrites in the prefrontal and higher-order visual cortices than in the primary visual cortex (Elston, 2003) might reflect such a functional difference. Alternatively, feedforward inhibition (cf. Troyer et al., 1998), which is not included in our model, might also be useful for the intensity invariance. Although which of them is, or whether both of them are, actually used requires future examinations, a potential advantage to use recurrent, rather than feedforward, inhibition would be efficiency; the same circuitry can be used for both competition and normalization in the case of global recurrent inhibition, or longdistance wiring is not necessary in the case of local recurrent inhibition. This might especially be the case for the prefrontal working memory circuit, which is considered to receive inputs from widely spread multiple brain regions.

Apart from the intensity invariance, contrast detection could be achieved not only by dendritic thresholding but also by somatic thresholding (i.e., just as a comparison between the strength of the external input and a positive somatic threshold). What is, if any, an advantage for the dendritic thresholding? If there is only a single threshold at the soma, recurrent excitation applied to a neuron always contributes to the neuronal output activity as long as the neuron is not completely silent. This appears to be not stable; once some neurons become slightly more active than the neighbors because of fluctuation, positive feedback inevitably occurs because of recurrent excitation so that the circuit converges to a winner(s)-take-all state. If there is dendritic thresholding, however, recurrent excitation can hardly boost the neuronal output activity unless it is indeed significant. Meanwhile, the cell can still fire at a low frequency, because of the current flow from some portion of the branches that receive strong external inputs and generate local spikes, so as to recruit recurrent inhibition, which in turn stabilizes such a low-activity state of the circuit.

\section{Possible link to animal behavior}

In the primate brain, spatial working memory circuits are considered to exist in the prefrontal cortex and also in other areas such as the parietal cortex or the superior colliculus. It has been observed that spatially tuned neural activity in those regions can be modulated by the saliency, uncertainty, or the context of the stimulus, as well as by the animal's attention or expectation (Basso and Wurtz, 1997; Sereno and Maunsell, 1998; Platt and 




B

a

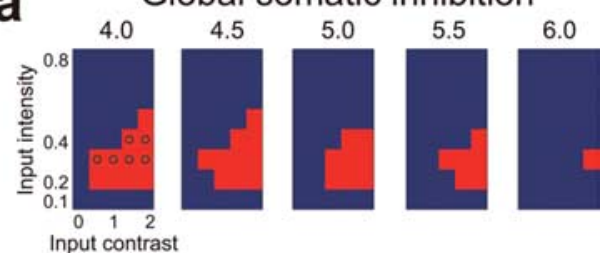

b



C With inter-branch heterogeneity \&
threshold for dendritic inhibition Local dendritic inhibition

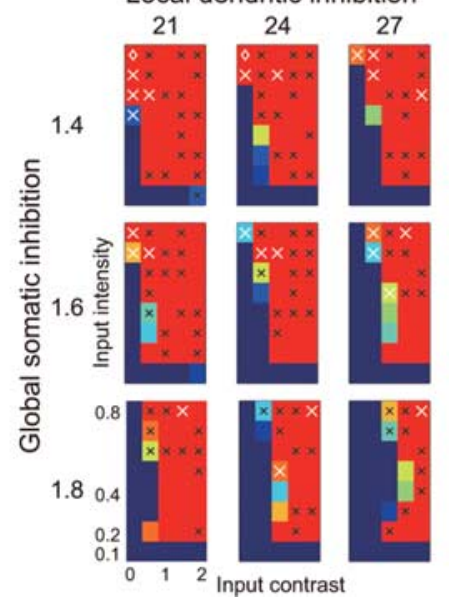

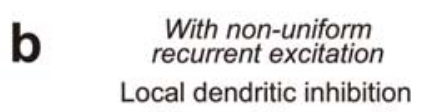

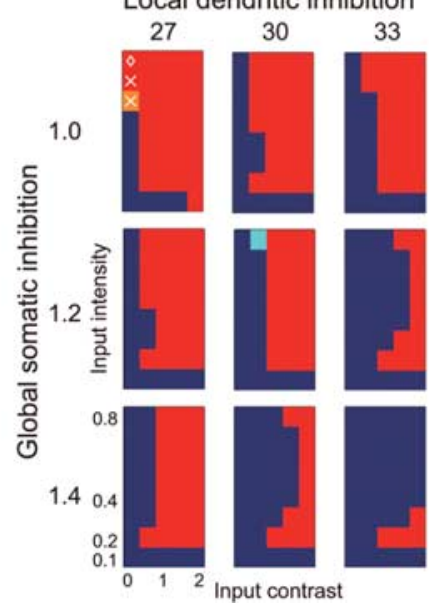

Figure 14. Behavior of the circuit when the input has time-varying noisy component (dynamic noise). $\boldsymbol{A a}, \boldsymbol{A b}$, The case with the moderately nonlinear dendrite and either global somatic recurrent inhibition ( $\boldsymbol{A} \boldsymbol{a})$ or global dendritic recurrent inhibition $(\boldsymbol{A} \boldsymbol{b})$. $\boldsymbol{A a}$, The solid line indicates the dependence of the memory accuracy on the input contrast (horizontal axis) in the static (time-invariant) input case (same as the solid line in Fig. 3C: averaged over 5000 trials). The crosses indicate the results for the case with dynamic noise (for details, see Materials and Methods) (averaged over 50 trials), looking comparable with the static input case. Memory was always formed in the both static and dynamic noise cases. The input intensity was 0.1. $\boldsymbol{A} \boldsymbol{b}$, The red line indicates the dependence of the probability of memory formation on the input contrast in the static input case (same as the red solid line in Fig. 4C:averaged over 5000 trials). The red dots indicate the results for the case with dynamic noise (averaged over 50 trials), looking similar to, but much steeper than the static input case. It seems reasonable: static noise-induced inhomogeneity of the neural activities could lead to memory formation through a stochastic resonancelike mechanism, whereas such an inhomogeneity would be temporally averaged out in the case with dynamic noise. The black line and the crosses (almost overlapped with the red dots on the top) indicate the memory accuracy in the cases with static or dynamic noise (averaged over 5000 or 50 trials), respectively. In both cases, memory was fairly accurate whenever it was formed. The inputintensity was $0.1 . \mathbf{B a}, \mathbf{B b}$, Probability of memory formation in the case with the highly nonlinear dendrite and either global somatic recurrent inhibition $(\mathbf{B a})$ or global dendritic recurrent inhibition $(\boldsymbol{B} \boldsymbol{b})$. The white crosses in $\boldsymbol{B} \boldsymbol{b}$ indicate $<0.98$ accuracy. The results look similar to the static input cases (Fig. $8 A, B$, respectively). $C a, C b$, Probability of memory formation in the case with the highly nonlinear dendrite, global somatic recurrent inhibition, and local dendritic recurrent inhibition whose range is 1.5 times wider than the feedforward and recurrent excitation (Fig. $11 \mathrm{Ac}$. The configurations are the same as in Figure 13. $(a$, The interbranch heterogeneity (Fig. $10 \mathrm{Ab}$ ) and the threshold for the recruitment of dendritic inhibition (0.05) were assumed in the same manner as Figure $13 C$. $C \boldsymbol{C}$, Recurrent excitation was assumed to be nonuniformly distributed over the branches (Fig. $1 G$, red dashed line) in the same manner as Figure $13 D$. In both cases $(\mathbf{C a}, \boldsymbol{C b})$, the results with dynamic noise look similar to those with static noise (Fig. 13C,D).

Glimcher, 1999; Constantinidis and Steinmetz, 2001; Bisley and Goldberg, 2003; Gold and Shadlen, 2007). Given that those areas are interconnected with each other, this would indicate that the input to the spatial working memory circuit can be modulated by those factors. An intriguing possibility is that the input contrast and the intensity (Fig. 2B) are modulated distinctively; for example, the contrast could represent the overall certainty of the stimulus location integrated from multiple sensory and mental modalities, whereas the intensity could reflect how many modalities were integrated or how long the integration lasted. Contrast-dependent intensityinsensitive accurate memory formation, enabled by dendritic compartmentalization as suggested in the present study, could then be useful for the animal to execute appropriate behavior, especially in a complex environment in which the animal needs to integrate information from multiple modalities. To verify the cellular mechanism proposed in the present study, it would be desired, although technically demanding, to measure the input to the pyramidal cell that shows sustained activity in the working memory task, which simulates such a complex environment, and how it varies according to the parameters of the task.

\section{Conflicting demands on neural modeling}

To make a neuron model closer to real neurons, there are two demands. One is the temporal aspect: how to describe the dynamics of action potential generation; the other, the spatial aspect: how to implement spatial extension of dendrites and linear and nonlinear processes thereon. For the simulation of large neuronal networks such as spatial working memory circuits, implementing both aspects is extremely difficult because of the limited computer power. Thus, although the description of firing dynamics has been drastically deepened from the rate-coding unit (Amari, 1977; Camperi and Wang, 1998; Fall and Rinzel, 2006) to the integrateand-fire model (Compte et al., 2000; Tanaka, 2002; Renart et al., 2003; Carter and Wang, 2007) and the conductancebased models (Gutkin et al., 2001; Tegnér et al., 2002; Wang et al., 2004), most studies have considered at most only a few serially connected compartments without branching. However, although there was an important indication that twocompartment models could well reproduce several phenomena from single neuronal firing patterns (Mainen and Sejnowski, 1996) to network rhythmogenesis (Pinsky and Rinzel, 1994), the recently established notion of the compartmentalized input integration in individual pyramidal dendritic branches cannot be explored by such models. The present study has implemented a complementary approach: modeling multiple dendritic branches while using 
simple rate-coding description. Such an approach to use "branching neuron model" as a component of the neural circuit has been proposed in several recent studies (Spratling and Johnson, 2001, 2002; Goldman et al., 2003; Morita and Aihara, 2005; Herz et al., 2006; Morita et al., 2007) and is expected to be applied to other systems.

Ideally, both the temporal and the spatial aspects should be taken into account at once. Features having slow time courses such as short-term synaptic plasticity could be incorporated under the current scheme of modeling. Because it is known that different interneuron subtypes show different short-term plasticity on their synapses (Reyes et al., 1998; Gibson et al., 1999), they are expected to be considered together in the model. Fast temporal features, namely, neuronal spiking should also matter, especially considering an indication that the degree of nonlinearity of the dendritic integration, which affected the circuit behavior in our model, would depend on the temporal and spatial profiles of the synaptic inputs (Gasparini and Magee, 2006). One possible direction is to consider a spiking neuron model with the minimum number of dendritic compartments. I have shown that a small number of branches are enough for reproducing at least some of the phenomena described in this study (Fig. 9). This would suggest the usefulness of considering a reduced spiking neuron model with a few parallel compartments, each of which receives inputs from different sources, instead of or in addition to widely considered tandem compartments.

\section{Experimentally testable predictions}

The model in this study would predict that when the spatial working memory circuit receives a low-contrast or background input, most dendritic branches of every pyramidal cell are below the threshold for dendritic spike generation so that they can hardly affect the somatic activity whereas a small portion of the branches do generate local spikes that make the cell fire at a low frequency (Figs. $5 A c, A d ; 7 A c, A d$, left; $B c, B d$, left), which in turn stabilizes the circuit by recruiting recurrent inhibition. When the circuit receives a high-contrast input, however, most dendritic branches of the pyramidal cell whose preference matches the presented stimulus are expected to receive suprathreshold inputs (Figs. $5 B c ; 7 A c$, right; $B c$, right). More precisely, those branches can be even at the saturated level, which would represent successive or prolonged dendritic spike generation. Recently, it was shown (Milojkovic et al., 2005) that if a single basal dendrite of the rat prefrontal pyramidal cell is stimulated strongly enough to generate a dendritic spike, the soma shows depolarization like the "UP" state (Steriade et al., 1993). Given that the in vitro UP state (Sanchez-Vives and McCormick, 2000; Shu et al., 2003a) would be an analog of the neuronal state in the awake animal and could correspond to both the high-level delay period activity and the low-level spontaneous firing, the results by Milojkovic et al. can be in line with the behavior of the present model. To further verify the validity of the model, it would be desired to test, in vitro or hopefully also in vivo, whether there exists such a correlation between the number/ratio of spike-generating branches and the neuronal firing rate as predicted above by simultaneously stimulating and/or recording multiple branches. At the macroscopic level, topographic organization of the spatial working memory circuits can now be visualized by functional magnetic resonance imaging (fMRI) in the parietal (Sereno et al., 2001; Schluppeck et al., 2005, 2006) and frontal (Kastner et al., 2007) cortices. Considering that the blood oxygen level-dependent signal of fMRI would be better correlated with the input to, rather than the output from, the neural population (Logothetis and Wandell,
2004), the central prediction of the present study that the working memory circuit possesses a potential to detect the signal-tonoise contrast of the external input, regardless of the absolute intensity, might become able to be tested, to some extent, by imaging studies in the future.

\section{References}

Amari S (1977) Dynamics of pattern formation in lateral-inhibition type neural fields. Biol Cybern 27:77-87.

Amari S, Arbib MA (1977) Competition and cooperation in neural nets. In: Systems neuroscience (Metzler J, ed), pp 119-165. New York: Academic

Archie KA, Mel BW (2000) A model for intradendritic computation of binocular disparity. Nat Neurosci 3:54-63.

Basso MA, Wurtz RH (1997) Modulation of neuronal activity by target uncertainty. Nature 389:66-69.

Beierlein M, Gibson JR, Connors BW (2003) Two dynamically distinct inhibitory networks in layer 4 of the neocortex. J Neurophysiol 90:2987-3000.

Bisley JW, Goldberg ME (2003) Neuronal activity in the lateral intraparietal area and spatial attention. Science 299:81-86.

Camperi M, Wang XJ (1998) A model of visuospatial working memory in prefrontal cortex: recurrent network and cellular bistability. J Comput Neurosci 5:383-405.

Carandini M, Heeger DJ (1994) Summation and division by neurons in primate visual cortex. Science 264:1333-1336.

Carter E, Wang XJ (2007) Cannabinoid-mediated disinhibition and working memory: dynamical interplay of multiple feedback mechanisms in a continuous attractor model of prefrontal cortex. Cereb Cortex 17 [Suppl 1]:i16-i26.

Cash S, Yuste R (1999) Linear summation of excitatory inputs by CA1 pyramidal neurons. Neuron 22:383-394.

Chance FS, Abbott LF, Reyes AD (2002) Gain modulation from background synaptic input. Neuron 35:773-782.

Compte A, Brunel N, Goldman-Rakic PS, Wang XJ (2000) Synaptic mechanisms and network dynamics underlying spatial working memory in a cortical network model. Cereb Cortex 10:910-923.

Constantinidis C, Steinmetz MA (2001) Neuronal responses in area 7a to multiple-stimulus displays: I. Neurons encode the location of the salient stimulus. Cereb Cortex 11:581-591.

Constantinidis C, Wang XJ (2004) A neural circuit basis for spatial working memory. Neuroscientist 10:553-565.

Constantinidis C, Williams GV, Goldman-Rakic PS (2002) A role for inhibition in shaping the temporal flow of information in prefrontal cortex. Nat Neurosci 5:175-180.

Elston GN (2003) Cortex, cognition and the cell: new insights into the pyramidal neuron and prefrontal function. Cereb Cortex 13:1124-1138.

Fall CP, Rinzel J (2006) An intracellular $\mathrm{Ca}^{2+}$ subsystem as a biologically plausible source of intrinsic conditional bistability in a network model of working memory. J Comput Neurosci 20:97-107.

Fukai T, Tanaka S (1997) A simple neural network exhibiting selective activation of neuronal ensembles: from winner-take-all to winners-share-all. Neural Comput 9:77-97.

Funahashi S, Bruce CJ, Goldman-Rakic PS (1989) Mnemonic coding of visual space in the monkey's dorsolateral prefrontal cortex. J Neurophysiol 61:331-349.

Galarreta M, Hestrin S (1998) Frequency-dependent synaptic depression and the balance of excitation and inhibition in the neocortex. Nat Neurosci 1:587-594.

Gao WJ, Wang Y, Goldman-Rakic PS (2003) Dopamine modulation of perisomatic and peridendritic inhibition in prefrontal cortex. J Neurosci 23:1622-1630.

Gasparini S, Magee JC (2006) State-dependent dendritic computation in hippocampal CA1 pyramidal neurons. J Neurosci 26:2088-2100.

Gibson JR, Beierlein M, Connors BW (1999) Two networks of electrically coupled inhibitory neurons in neocortex. Nature 402:75-79.

Gold JI, Shadlen MN (2007) The neural basis of decision making. Annu Rev Neurosci 30:535-574.

Golding NL, Staff NP, Spruston N (2002) Dendritic spikes as a mechanism for cooperative long-term potentiation. Nature 418:326-331.

Goldman MS, Maldonado P, Abbott LF (2002) Redundancy reduction and sustained firing with stochastic depressing synapses. J Neurosci 22:584-591. 
Goldman MS, Levine JH, Major G, Tank DW, Seung HS (2003) Robust persistent neural activity in a model integrator with multiple hysteretic dendrites per neuron. Cereb Cortex 13:1185-1195.

Goldman-Rakic PS (1995) Cellular basis of working memory. Neuron 14:477-485.

González-Burgos G, Krimer LS, Urban NN, Barrionuevo G, Lewis DA (2004) Synaptic efficacy during repetitive activation of excitatory inputs in primate dorsolateral prefrontal cortex. Cereb Cortex 14:530-542.

González-Burgos G, Krimer LS, Povysheva NV, Barrionuevo G, Lewis DA (2005) Functional properties of fast spiking interneurons and their synaptic connections with pyramidal cells in primate dorsolateral prefrontal cortex. J Neurophysiol 93:942-953.

Govindarajan A, Kelleher RJ, Tonegawa S (2006) A clustered plasticity model of long-term memory engrams. Nat Rev Neurosci 7:575-583.

Gupta A, Wang Y, Markram H (2000) Organizing principles for a diversity of GABAergic interneurons and synapses in the neocortex. Science 287:273-278.

Gutkin BS, Laing CR, Colby CL, Chow CC, Ermentrout GB (2001) Turning on and off with excitation: the role of spike-timing asynchrony and synchrony in sustained neural activity. J Comput Neurosci 11:121-134.

Hahnloser RH, Sarpeshkar R, Mahowald MA, Douglas RJ, Seung HS (2000) Digital selection and analogue amplification coexist in a cortex-inspired silicon circuit. Nature 405:947-951.

Harvey CD, Svoboda K (2007) Locally dynamic synaptic learning rules in pyramidal neuron dendrites. Nature 450:1195-1200.

Häusser M, Mel BW (2003) Dendrites: bug or feature? Curr Opin Neurobiol 13:372-383.

Herz AV, Gollisch T, Machens CK, Jaeger D (2006) Modeling single-neuron dynamics and computations: a balance of detail and abstraction. Science 314:80-85.

Hô N, Destexhe A (2000) Synaptic background activity enhances the responsiveness of neocortical pyramidal neurons. J Neurophysiol 84:1488-1496.

Holt GR, Koch C (1997) Shunting inhibition does not have a divisive effect on firing rates. Neural Comput 9:1001-1013.

Jadi M, Mel BW (2007) Synchrony and location dependent effects of inhibition in a pyramidal neuron model. Paper presented at the $2007 \mathrm{Com}$ putational and Systems Neuroscience Meeting, Salt Lake City, UT, February.

Kapfer C, Glickfeld LL, Atallah BV, Scanziani M (2007) Supralinear increase of recurrent inhibition during sparse activity in the somatosensory cortex. Nat Neurosci 10:743-753.

Kastner S, DeSimone K, Konen CS, Szczepanski SM, Weiner KS, Schneider KA (2007) Topographic maps in human frontal cortex revealed in memory-guided saccade and spatial working-memory tasks. J Neurophysiol 97:3494-3507.

Kawaguchi Y, Kubota Y (1997) GABAergic cell subtypes and their synaptic connections in rat frontal cortex. Cereb Cortex 7:476-486.

Koch C, Poggio T, Torre V (1983) Nonlinear interactions in a dendritic tree: localization, timing, and role in information processing. Proc Natl Acad Sci U S A 80:2799-2802.

Kubota Y, Hatada S, Kondo S, Karube F, Kawaguchi Y (2007) Neocortical inhibitory terminals innervate dendritic spines targeted by thalamocortical afferents. J Neurosci 27:1139-1150.

Liu G (2004) Local structural balance and functional interaction of excitatory and inhibitory synapses in hippocampal dendrites. Nat Neurosci 7:373-379.

Logothetis NK, Wandell BA (2004) Interpreting the BOLD signal. Annu Rev Physiol 66:735-769.

London M, Häusser M (2005) Dendritic computation. Annu Rev Neurosci 28:503-532.

Losonczy A, Magee JC (2006) Integrative properties of radial oblique dendrites in hippocampal CA1 pyramidal neurons. Neuron 50:291-307.

Losonczy A, Makara JK, Magee JC (2008) Compartmentalized dendritic plasticity and input feature storage in neurons. Nature 452:436-441.

Mainen ZF, Sejnowski TJ (1996) Influence of dendritic structure on firing pattern in model neocortical neurons. Nature 382:363-366.

Mainen ZF, Joerges J, Huguenard JR, Sejnowski TJ (1995) A model of spike initiation in neocortical pyramidal neurons. Neuron 15:1427-1439.

Markram H, Toledo-Rodriguez M, Wang Y, Gupta A, Silberberg G, Wu C (2004) Interneurons of the neocortical inhibitory system. Nat Rev Neurosci 5:793-807.
Matsuzaki M (2007) Factors critical for the plasticity of dendritic spines and memory storage. Neurosci Res 57:1-9.

Mehta MR (2004) Cooperative LTP can map memory sequences on dendritic branches. Trends Neurosci 27:69-72.

Mel BW (1993) Synaptic integration in an excitable dendritic tree. J Neurophysiol 70:1086-1101.

Mel BW (2007) Why have dendrites? A computational perspective. In: Dendrites, Ed 2 (Stuart GJ, Spruston N, Häusser M, eds), pp 351-399. Oxford: Oxford UP.

Mel BW, Ruderman DL, Archie KA (1998) Translation-invariant orientation tuning in visual "complex" cells could derive from intradendritic computations. J Neurosci 18:4325-4334.

Milojkovic BA, Radojicic MS, Antic SD (2005) A strict correlation between dendritic and somatic plateau depolarizations in the rat prefrontal cortex pyramidal neurons. J Neurosci 25:3940-3951.

Milojkovic BA, Zhou WL, Antic SD (2007) Voltage and calcium transients in basal dendrites of the rat prefrontal cortex. J Physiol 585:447-468.

Morita K, Aihara K (2005) A network model with pyramidal cells and GABAergic non-FS cells in the cerebral cortex. Neurocomputing 65:697-707.

Morita K, Amari S-I (2007) A field equation model of the neuronal network dynamics incorporating two stages of nonlinear input integrations in individual neurons. Soc Neurosci Abstr 33:753.6.

Morita K, Okada M, Aihara K (2007) Selectivity and stability via dendritic nonlinearity. Neural Comput 19:1798-1853.

Nevian T, Larkum ME, Polsky A, Schiller J (2007) Properties of basal dendrites of layer 5 pyramidal neurons: a direct patch-clamp recording study. Nat Neurosci 10:206-214.

Pinsky PF, Rinzel J (1994) Intrinsic and network rhythmogenesis in a reduced Traub model for CA3 neurons. J Comput Neurosci 1:39-60.

Platt ML, Glimcher PW (1999) Neural correlates of decision variables in parietal cortex. Nature 400:233-238.

Poirazi P, Mel BW (2001) Impact of active dendrites and structural plasticity on the memory capacity of neural tissue. Neuron 29:779-796.

Poirazi P, Brannon T, Mel BW (2003a) Arithmetic of subthreshold synaptic summation in a model CA1 pyramidal cell. Neuron 37:977-987.

Poirazi P, Brannon T, Mel BW (2003b) Pyramidal neuron as two-layer neural network. Neuron 37:989-999.

Polsky A, Mel BW, Schiller J (2004) Computational subunits in thin dendrites of pyramidal cells. Nat Neurosci 7:621-627.

Prescott SA, De Koninck Y (2003) Gain control of firing rate by shunting inhibition: roles of synaptic noise and dendritic saturation. Proc Natl Acad Sci U S A 100:2076-2081.

Rall W (1964) Theoretical significance of dendritic trees for neuronal input-output relations. In: Neural theory and modeling (Reiss RF, ed), pp 73-97. Stanford, CA: Stanford UP.

Rao SG, Williams GV, Goldman-Rakic PS (1999) Isodirectional tuning of adjacent interneurons and pyramidal cells during working memory: evidence for microcolumnar organization in PFC. J Neurophysiol 81:1903-1916.

Rao SG, Williams GV, Goldman-Rakic PS (2000) Destruction and creation of spatial tuning by disinhibition: GABA $_{\mathrm{A}}$ blockade of prefrontal cortical neurons engaged by working memory. J Neurosci 20:485-494.

Renart A, Song P, Wang XJ (2003) Robust spatial working memory through homeostatic synaptic scaling in heterogeneous cortical networks. Neuron 38:473-485.

Reyes A, Lujan R, Rozov A, Burnashev N, Somogyi P, Sakmann B (1998) Target-cell-specific facilitation and depression in neocortical circuits. Nat Neurosci 1:279-285.

Rhodes P (2006) The properties and implications of NMDA spikes in neocortical pyramidal cells. J Neurosci 26:6704-6715.

Sanchez-Vives MV, McCormick DA (2000) Cellular and network mechanisms of rhythmic recurrent activity in neocortex. Nat Neurosci 3:1027-1034.

Schiller J, Major G, Koester HJ, Schiller Y (2000) NMDA spikes in basal dendrites of cortical pyramidal neurons. Nature 404:285-289.

Schluppeck D, Glimcher P, Heeger DJ (2005) Topographic organization for delayed saccades in human posterior parietal cortex. J Neurophysiol 94:1372-1384.

Schluppeck D, Curtis CE, Glimcher PW, Heeger DJ (2006) Sustained activity in topographic areas of human posterior parietal cortex during memory-guided saccades. J Neurosci 26:5098-5108. 
Segev I (1995) Dendritic processing. In: The handbook of brain theory and neuronal networks (Arbib M, ed), pp 282-289. Cambridge, MA: MIT.

Segev I, Parnas I (1983) Synaptic integration mechanisms. Theoretical and experimental investigation of temporal postsynaptic interactions between excitatory and inhibitory inputs. Biophys J 41:41-50.

Sereno AB, Maunsell JH (1998) Shape selectivity in primate lateral intraparietal cortex. Nature 395:500-503.

Sereno MI, Pitzalis S, Martinez A (2001) Mapping of contralateral space in retinotopic coordinates by a parietal cortical area in humans. Science 294:1350-1354.

Shepherd GM, Brayton RK (1987) Logic operations are properties of computer-simulated interactions between excitable dendritic spines. Neuroscience 21:151-165.

Shu Y, Hasenstaub A, McCormick DA (2003a) Turning on and off recurrent balanced cortical activity. Nature 423:288-293.

Shu Y, Hasenstaub A, Badoual M, Bal T, McCormick DA (2003b) Barrages of synaptic activity control the gain and sensitivity of cortical neurons. J Neurosci 23:10388-10401.

Silberberg G, Markram H (2007) Disynaptic inhibition between neocortical pyramidal cells mediated by Martinotti cells. Neuron 53:735-746.

Somogyi P, Tamás G, Lujan R, Buhl EH (1998) Salient features of synaptic organisation in the cerebral cortex. Brain Res Brain Res Rev 26:113-135.

Spratling MW, Johnson MH (2001) Dendritic inhibition enhances neural coding properties. Cereb Cortex 11:1144-1149.

Spratling MW, Johnson MH (2002) Preintegration lateral inhibition enhances unsupervised learning. Neural Comput 14:2157-2179.

Spruston N (2008) Pyramidal neurons: dendritic structure and synaptic integration. Nat Rev Neurosci 9:206-221.

Spruston N, Stuart GJ, Häusser M (2007) Dendritic integration. In: Dendrites, Ed 2 (Stuart GJ, Spruston N, Häusser M, eds), pp 351-399. Oxford: Oxford UP.
Steriade M, Nuñez A, Amzica F (1993) A novel slow ( $<1 \mathrm{~Hz}$ ) oscillation of neocortical neurons in vivo: depolarizing and hyperpolarizing components. J Neurosci 13:3252-3265.

Tamás G, Buhl EH, Somogyi P (1997) Fast IPSPs elicited via multiple synaptic release sites by different types of GABAergic neurone in the cat visual cortex. J Physiol 500:715-738.

Tanaka S (2002) Dopamine controls fundamental cognitive operations of multi-target spatial working memory. Neural Netw 15:573-582.

Tegnér J, Compte A, Wang XJ (2002) The dynamical stability of reverberatory neural circuits. Biol Cybern 87:471-481.

Troyer TW, Krukowski AE, Priebe NJ, Miller KD (1998) Contrast-invariant orientation tuning in cat visual cortex: thalamocortical input tuning and correlation-based intracortical connectivity. J Neurosci 18:5908-5927.

Wang XJ, Tegnér J, Constantinidis C, Goldman-Rakic PS (2004) Division of labor among distinct subtypes of inhibitory neurons in a cortical microcircuit of working memory. Proc Natl Acad Sci U S A 101:1368-1373.

Wang Y, Gupta A, Toledo-Rodriguez M, Wu CZ, Markram H (2002) Anatomical, physiological, molecular and circuit properties of nest basket cells in the developing somatosensory cortex. Cereb Cortex 12:395-410.

Wei DS, Mei YA, Bagal A, Kao JP, Thompson SM, Tang CM (2001) Compartmentalized and binary behavior of terminal dendrites in hippocampal pyramidal neurons. Science 293:2272-2275.

Williams SR (2004) Spatial compartmentalization and functional impact of conductance in pyramidal neurons. Nat Neurosci 7:961-967.

Xie X, Hahnloser RH, Seung HS (2002) Selectively grouping neurons in recurrent networks of lateral inhibition. Neural Comput 14:2627-2646.

Zaitsev AV, Gonzalez-Burgos G, Povysheva NV, Kröner S, Lewis DA, Krimer LS (2005) Localization of calcium-binding proteins in physiologically and morphologically characterized interneurons of monkey dorsolateral prefrontal cortex. Cereb Cortex 15:1178-1186. 